\title{
WAFER-LEVEL VACUUM-ENCAPSULATED ULTRA-LOW VOLTAGE TUNING FORK MEMS RESONATOR
}

\author{
Thesis \\ Submitted to \\ The School of Engineering of the \\ UNIVERSITY OF DAYTON \\ In Partial Fulfillment of the Requirements for \\ The Degree of \\ Master of Science in Electrical Engineering
}

By

Junjun Huan

UNIVERSITY OF DAYTON

Dayton, Ohio

May 2017 


\section{WAFER-LEVEL VACUUM-ENCAPSULATED ULTRA-LOW VOLTAGE TUNING FORK MEMS RESONATOR}

Name: Huan, Junjun

APPROVED BY:

Vamsy Chodavarapu

Advisory Committee Chairman

Associate Professor

Department of Electrical and Computer

Engineering
Guru Subramanyam

Committee Member

Professor

Department of Electrical and Computer

Engineering

Weisong Wang

Committee Member

Research Engineer

Department of Electrical and

Computer Engineering

Robert J. Wilkens, Ph.D., P.E.

Associate Dean for Research and Innovation

Professor

School of Engineering
Eddy M. Rojas, Ph.D., M.A., P.E.

Dean

School of Engineering 


\section{CCopyright by}

Junjun Huan

All rights reserved

2017 


\section{ABSTRACT \\ WAFER-LEVEL VACUUM-ENCAPSULATED ULTRA-LOW \\ VOLTAGE TUNING FORK MEMS RESONATOR}

Name: Junjun Huan

University of Dayton

Advisor: Vamsy Chodavarapu

In this thesis, a low-voltage $32 \mathrm{kHz}$ silicon tuning fork MicroElectroMechanical Systems (MEMS)-based resonator design with a high Quality factor of over 73,000 is presented with a Complementary Metal-Oxide Semiconductor (CMOS) sustaining amplifier towards a low power oscillator. The resonator is designed using MEMS Integrated Design for Inertial Sensors (MIDIS) process developed by Teledyne DALSA Semiconductor Inc. (TDSI). MIDIS offers wafer-level vacuum encapsulation with ultra-low leak rate. Ultra-low polarization voltage, as low as $10 \mathrm{mV}$, is needed to excite the resonator by using a transduction gap reduction technique based on electrostatic deflection of movable electrodes and subsequent localized melting of welding pads for permanent position locking. Further, the technique helps to minimize unexpected electrostatic stiffness induced by time-varying capacitance across transduction gaps to just -0.6 $\mathrm{N} / \mathrm{m}$. The motional resistance drops down to about $2 \mathrm{k} \Omega$ as a result of a small gap size and the technique helps to improve the Quality Factor (Q). A sustaining amplifier using a transimpedance operational amplifier configuration is system-integrated with the tuning fork resonator to establish continuous oscillation with low damping losses. An average power consumption of around $600 \mu \mathrm{W}$ is measured on the oscillator, which is suitable for mobile electronic systems. 
Dedicated to my parents 


\section{ACKNOWLEDGEMENTS}

My special thanks to Dr. Vamsy Chodavarapu, my advisor, for giving me guidance all through my research work as well as unlimited access to the software and other resources necessary for completing the design of this work.

I would also like to appreciate everyone who has offered me assistance in this work. This includes George Xereas, who spared some of his valuable time to teach me with patience how to use the CoventorWare software for MEMS-based resonator design; Balaadithya Uppalapati, who gave me the basic tutorials on how Silvaco EDA tools should be used for oscillator circuit design; and Dr. Weisong Wang, for imparting knowledge of MEMS fundamentals to me through lectures in class. I also deeply appreciate Dr. Guru Subramanyam, who provided me with several useful points on which parts I should improve regarding the work in the future. 


\section{TABLE OF CONTENTS}

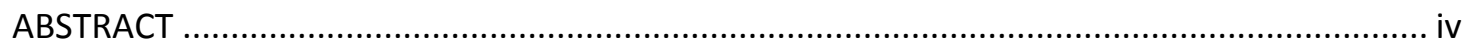

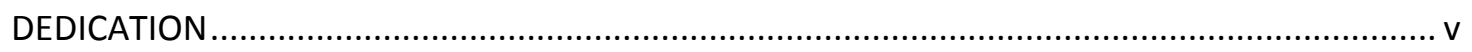

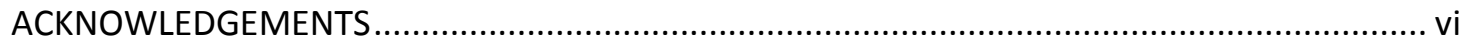

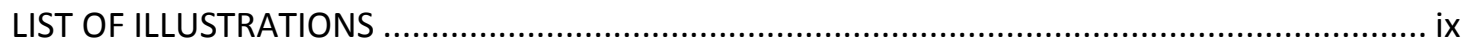

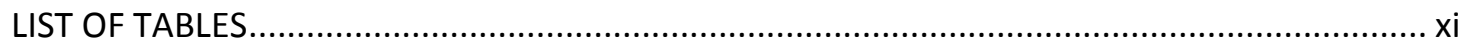

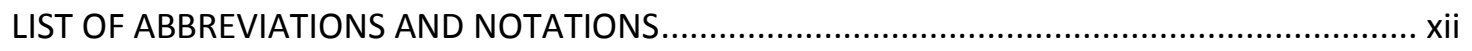

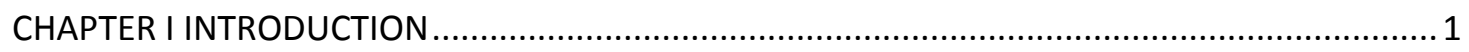

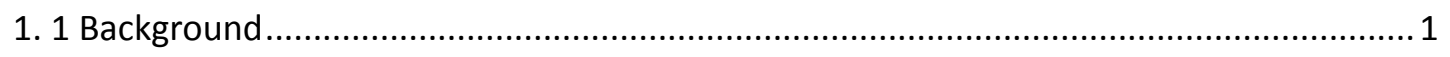

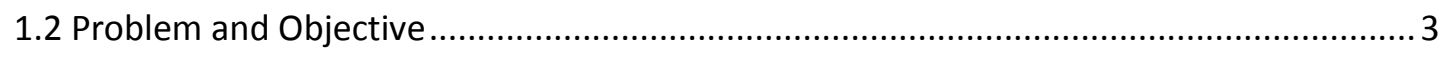

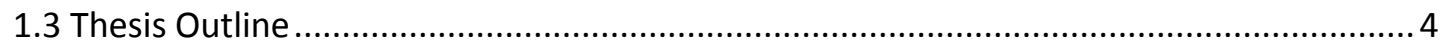

CHAPTER II MIDIS FABRICATION PROCESS ARCHITECTURE.............................................. 5

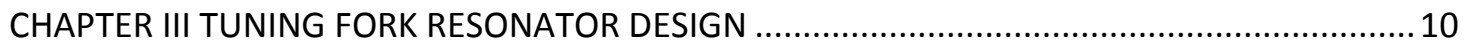

3.1 Transduction Gap Reduction Technology .................................................................... 11

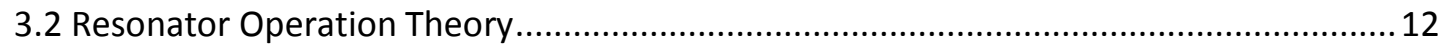

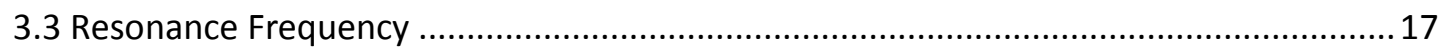

3.4 Frequency Softening Induced by Electrostatic Stiffness ............................................... 19

3.5 Energy Loss Evaluation (Quality Factor) .................................................................. 21

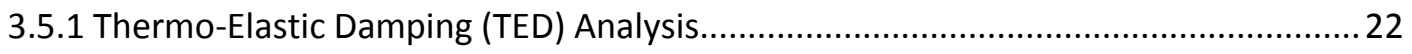

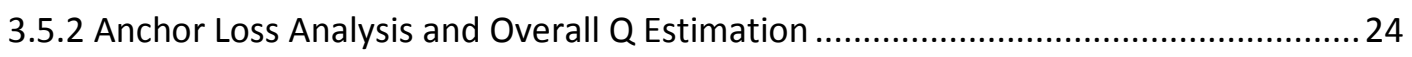

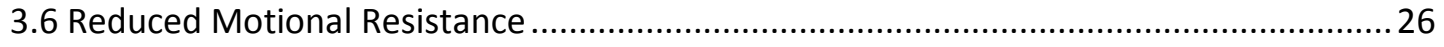


4.1 Electrical Equivalent Circuit Model of the Resonator

4.2 CMOS Transimpedance Operational Amplifier ............................................................... 30

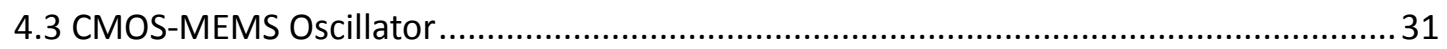

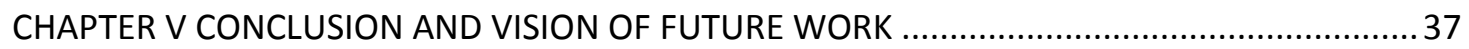

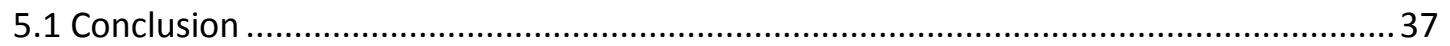

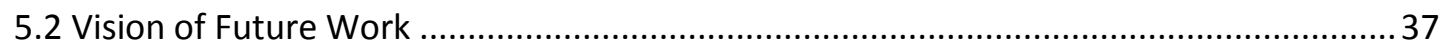

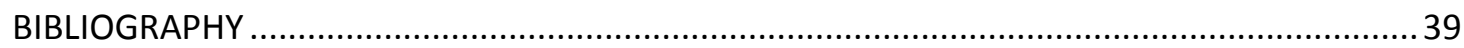

APPENDIX A. Harmonic Current of the Resonator with Different Transduction Gap Sizes...... 41 


\section{LIST OF ILLUSTRATIONS}

Figure 1. Cross-Sectional View of the 3D Resonator in MIDIS Process........................................ 7

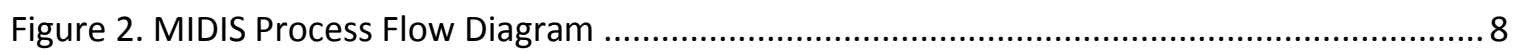

Figure 3. Tuning Fork Resonator Device Structure ........................................................... 11

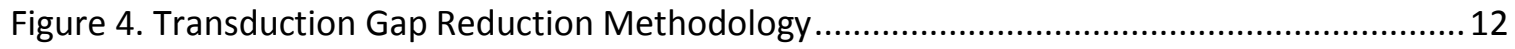

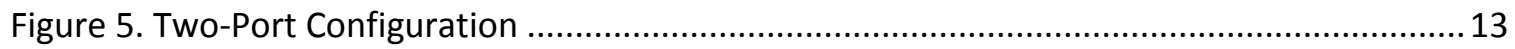

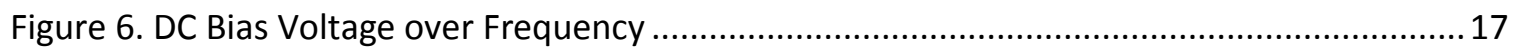

Figure 7. Harmonic Current Frequency Response ................................................................. 18

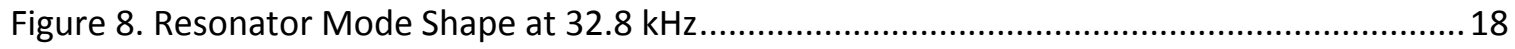

Figure 9. Softened Frequency over Transduction Gap Size .................................................... 21

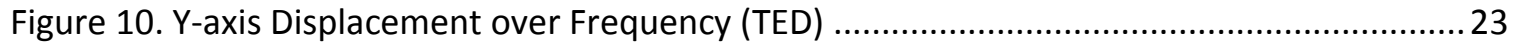

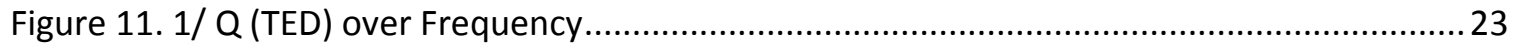

Figure 12. Y-axis Displacement Over Frequency (Anchor Loss) ................................................ 25

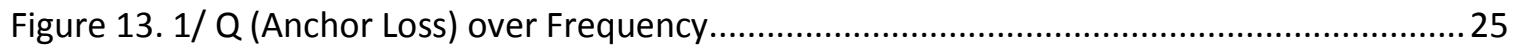

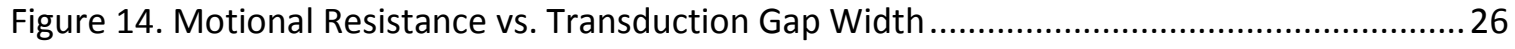

Figure 15. Electrical Equivalent Circuit Model .................................................................... 29

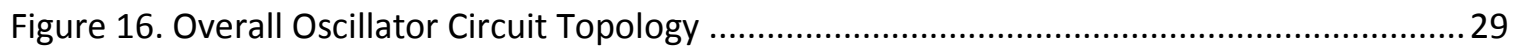

Figure 17. Physical Layout of the Operational Amplifier Circuit.................................................. 31

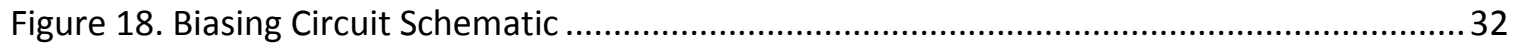

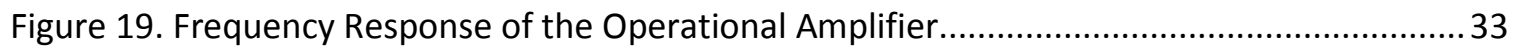


Figure 20. Frequency Response of the Oscillator

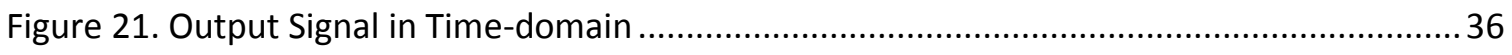

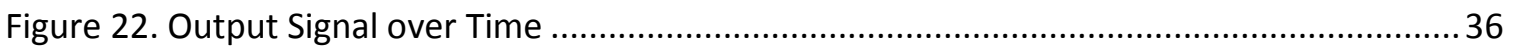

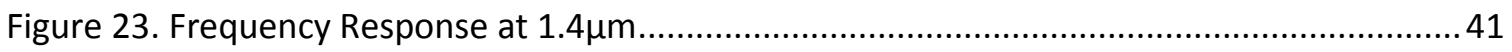

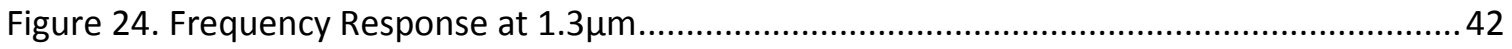

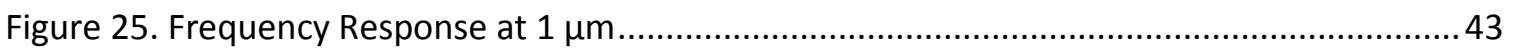

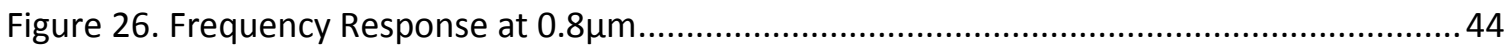

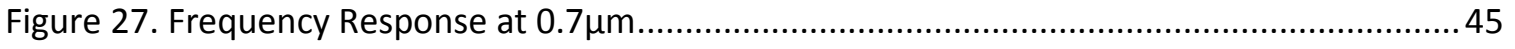

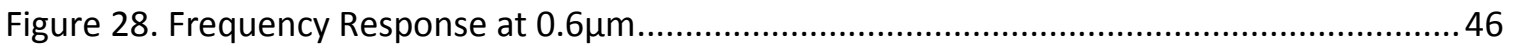

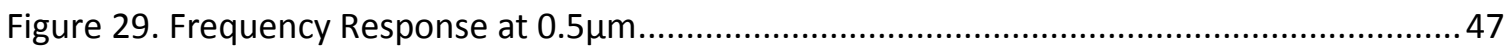

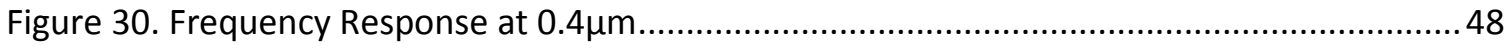

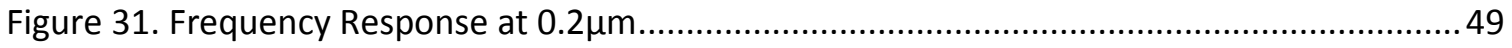

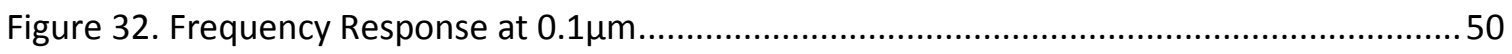

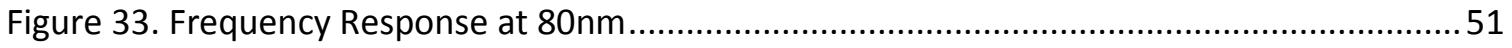




\section{LIST OF TABLES}

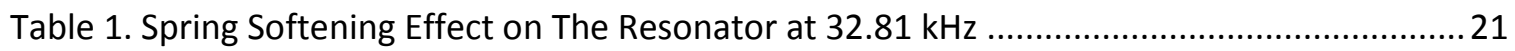

Table 2. Transistor Parameters of The Transimpedance Operational Amplifier ...........................33

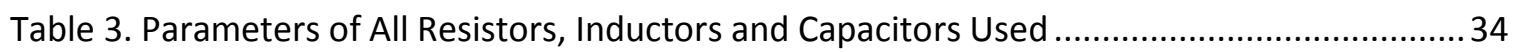

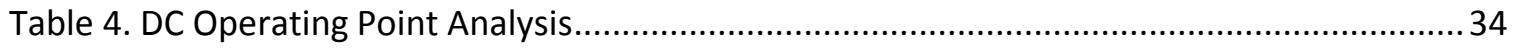




\title{
LIST OF ABBREVIATIONS AND NOTATIONS
}

\author{
A \\ $A_{o}$ \\ $\mathrm{AC}$ \\ $c_{e}$ \\ $C_{c}$ \\ CMOS \\ $C_{0}$ \\ $C_{x}$ \\ $C_{p}$ \\ DC \\ DRIE \\ $F_{E}$ \\ FEM \\ $F_{i}$ \\ $f_{0}$ \\ $f_{\text {re }}$ \\ $\mathrm{GF}$ \\ $g_{0}$ \\ $\mathrm{H}$ \\ $\mathrm{H}_{2} \mathrm{O}$ \\ IF \\ IOT \\ $i_{o}$ \\ $I_{S}$ \\ $I_{\text {ssum }}$ \\ ISDP \\ $k_{e}$ \\ $k_{m}$ \\ $k_{r e}$ \\ $\mathrm{KOH}$ \\ L \\ $\mathrm{L}_{x}$ \\ $m_{e}$ \\ MIDIS \\ N \\ $P_{E}$ \\ PECVD \\ ppm \\ Overlapping Area Between One Resonant and Electrode Finger Pair \\ Overall Overlapping Area Between Resonant and Electrode Fingers \\ Alternate Current \\ Damping Coefficient \\ Capacitance for Operational Amplifier Compensation \\ Complementary Metal-Oxide Semiconductor \\ Static Capacitance across Each Electrode and Resonant Finger Pair \\ Motional Capacitance \\ Parasitic capacitance \\ Direct Current \\ Deep Reactive-Ion Etching Method \\ Electrostatic Force \\ Finite Element Modeling \\ External Input force \\ Natural Resonance Frequency \\ Softened Resonance Frequency \\ Global Foundry \\ Initial Transduction Gap Width \\ Height \\ Water Molecule \\ Intermediate Frequency \\ Internet -of-Things \\ Output Current \\ DC Source Current of a MOSFET Device \\ Sum of Current Sourced From Power Supply \\ In-Situ Doped Polysilicon \\ Electrostatic Stiffness of a Spring System \\ Mechanical Stiffness of a Spring System \\ Effective Stiffness of a Spring System \\ Potassium Hydroxide \\ Length \\ Motional Inductance \\ Effective Mass of The Resonator \\ MEMS Integrated Design for Inertial Sensors \\ Number of Comb Fingers \\ Electrostatic Pressure \\ Plasma Enhanced Chemical Vapor Deposition \\ Part Per Million
}




$\begin{array}{cc}\mathrm{Q} & \text { Quality Factor } \\ Q_{\text {Anchor }} & \text { Anchor Loss Quality Factor } \\ Q_{\text {Gas }} & \text { Air Damping Quality Factor } \\ Q_{\text {Others }} & \text { Quality Factor of Other Energy Loss Mechanism } \\ Q_{\text {TED }} & \text { Thermo-Elastic Damping Quality Factor } \\ Q_{\text {Total }} & \text { Total Quality Factor } \\ \mathrm{REF} . & \text { Reference } \\ \mathrm{RF} & \text { Radio Frequency } \\ \mathrm{R}_{x} & \text { Motional Resistance } \\ \mathrm{SCS} & \text { Single Crystal Silicon } \\ \mathrm{SiO} \mathrm{O}_{2} & \text { Silicon Dioxide } \\ \mathrm{Si}(\mathrm{OC} 2 \mathrm{H} 5) 4 & \text { Tetraethyl Orthosilicate } \\ \mathrm{t} & \text { Thickness } \\ \mathrm{TDSI} & \text { Teledyne DALSA Semiconductor Inc. } \\ \mathrm{TSV} & \text { Through Silicon Via } \\ V_{D D} & \text { DC Power Supply } \\ V_{i} & \text { Input Voltage } \\ V_{p} & \text { DC polarization voltage } \\ \mathrm{W} & \text { Width } \\ w & \text { Angular Frequency } \\ w_{0} & \text { Capacitance over Displacement Ratio at port } \mathrm{n} \\ x & \text { Dielectric Constant of Free Space } \\ \dot{x} & \text { Angular Frequency at Resonance } \\ \ddot{x} & \text { Displacement of The Resonator } \\ \partial w & \text { The First Derivative of } \\ \partial C_{n} & x \\ \eta_{e n} & x \\ & \text { The Second Derivative of } \\ & x\end{array}$




\section{CHAPTER I}

\section{INTRODUCTION}

\section{1 Background}

Almost every electronic system has more than one oscillator built inside for frequency reference or timing control purpose [1, 2]. Those applications include real time clocks, frequency conversion in RF transceiver architectures, providing clock signals to synchronize states of different devices on sequential logic circuits and frequency reference for data transmission [2]. Currently, oscillators on the market can be divided into two families, mechanical and electrical oscillators. Mechanical oscillators such as quartz crystal or ceramic oscillators, are capable of achieving high $Q$ of nearly 10,000 [1] and are widely used for Radio Frequency (RF) and Intermediate Frequency (IF) filters on RF front-end circuits. However, they cannot be monolithically integrated with CMOS Integrated Circuits (ICS). In contrast, electrical or electronic oscillators such digital ring resonators or circuit tanks of resistors, capacitors and inductors (RCL) as frequency selective elements are monolithically integrated with CMOS ICs, but at the expense

of low $Q$ and poor frequency stability [2]. Now an emerging MicroElectroMechanical Systems (MEMS) technology based on mechanical oscillators enables high-Q along with single-chip integration with CMOS circuitry. These micromechanical components, mostly MEMS resonators are based on piezoelectric or electrostatic actuation and help to meet the requirements on low 
cost, low power consumption and small motional resistance imposed by mobile or wearable technology and Internet -of -Things (IOT) applications.

Low frequency oscillators at $32.768 \mathrm{kHz}$ are widely used in many mobile or wearable electronic systems. In these applications, power consumption has to be extremely low to allow battery powered devices to operate for long duration. In most battery-powered devices, battery life can be preserved by minimizing the amount of current needed to drive the electronic systems. The frequency value of " $32.768 \mathrm{kHz}$ ", is a result of $2^{15}$ and can be conveniently downconverted to frequency of 1 cycle per period (or $1 \mathrm{~Hz}$ ) through a 15-bit binary counter typically functioned by a chain of flip-flops or latches.

To benefit from technology scaling with advantages such as fast speed, low power consumption and high-level system integration [1], there has been significant interests towards MEMS-based oscillators that provide $32 \mathrm{kHz}$ output [3-6]. Many previous research studies focused on lowering total power consumption, scaling down device size, minimizing phase noise and improving frequency accuracy and stability over time, since the first generation of $32 \mathrm{kHz}$ MEMS oscillator emerged in 2005 [3-6]. The first MEMS-based oscillator was designed to consume only $1 \mu \mathrm{W}$ power, which was on par with that of its contemporary quartz crystal competitors. Besides, laser trimming technology was used to correct frequency offset[3]. In 2011, the first tunable piezoelectric MEMS Resonators operating at $32.768 \mathrm{kHz}$ was designed to compensate large Temperature Coefficient of Frequency (TCF) of about $-30 \mathrm{ppm} /{ }^{\circ} \mathrm{C}$ across temperature from $-50^{\circ} \mathrm{C}$ to $100^{\circ} \mathrm{C}$ by using a tuning voltage of $4 \mathrm{~V}$ [4]. Later in 2012 , a new design of electrostatic $32 \mathrm{kHz}$ MEMS-based oscillator was presented. The resonator device was measured to occupy a die area of just $0.0154 \mathrm{~mm}^{2}$, which was a huge breakthrough among all compact-footprint clock oscillators design presented at that frequency range. In addition, voltage-control frequency tuning technology was used to allow for a 50,000 ppm frequency 
tuning, greatly improving frequency accuracy and stability over time [5]. Then three years later, two popular frequency control methods of phase lock loop and temperature compensated ppm circuits were combined and added into a commercially available $32 \mathrm{kHz}$ MEMS-based oscillator. The design results in frequency stability calibrated to be just $\pm 3 \mathrm{ppm}[6]$.

\subsection{Problem and Objective}

While providing many advantages compared to mechanical and piezoelectric competitors, electrostatic MEMS-based resonators face a bottleneck with requiring a relatively high polarization voltage. The high polarization voltage is mainly dependent on the transduction gap size which ultimately depends on the capabilities of the fabrication process. Further, electrostatic MEMS-based resonators require a good vacuum encapsulation with low leak rate to obtain a high Quality Factor (Q) from the resonator performance by minimizing the damping losses. A large transduction gap also leads to a large motional resistance, increased power consumption and low power handling ability of the resonators.

This thesis is thereby aiming to study the application of a novel transduction gap reduction technology [7] to a design of $32 \mathrm{kHz}$ oscillator using a wafer-level vacuumencapsulated silicon electrostatic tuning fork resonator. For the same resonator design with reducing the transduction gap to different values, we will study the performance on DC polarization voltage, motional resistance and electrostatic stiffness. An optimized transduction gap size will be sorted out in terms of the results. As $Q$ is a key consideration, micromechanical resonator will be designed to achieve a high $Q$ which will lead to improved spectral purity. Our MEMS resonator is customized to be designed in MEMS Integrated Design for Inertial Sensors $\left(\right.$ MIDIS $^{\mathrm{TM}}$ ) process from Teledyne DALSA Semiconductor Incorporation (TDSI). It is an ultra-clean

vacuum sealing process, which enables a high $Q$ factor for resonators [8] with minimizing damping losses. In this thesis, the MEMS-based resonator is also used as a frequency selective 
element to be integrated with a sustaining high gain and low power CMOS operational amplifier to form a complete oscillator circuit.

\subsection{Thesis Outline}

In chapter II, the fabrication and encapsulation process flow, MIDIS process from TDSI used for our MEMS-based resonator design will be described in detail. Also key features and benefits of the process will be discussed.

Chapter III will describe our silicon tuning fork resonator design. First, features of main components on the device structure will be described. Then, the working mechanism of the device will be discussed in detail. Later, the transduction gap size of our resonator is reduced using gap reduction methodology with welding pads and this gap reduction process will be explained. The effect of the gap reduction method on our resonator design will be studied through simulations of the resonator design with different gap sizes.

Chapter IV will describe the MEMS-CMOS oscillator circuit design. It involves a discussion on electrical equivalent circuit of the MEMS resonator as a frequency selective tank, the high gain sustaining transimpedance amplifier and the system-integration between these two to function as an oscillator. Apart from the circuit schematic design, this chapter will also depict a layout design of the operational amplifier using custom layout process design kit form Global Foundry (GF) Company.

Chapter V will conclude the thesis with a brief summary and vision for future work. 


\section{CHAPTER II \\ MIDIS FABRICATION PROCESS ARCHITECTURE}

MEMS Integrated Design for Inertial Sensors (MIDIS) is a platform developed by Teledyne DALSA Semiconductor Incorporated (TDSI) for customers to design motion sensors using a wafer-level vacuum encapsulation packaging [8]. Figure 1 depicts the cross-sectional view of our MEMS-based resonator designed in MIDIS process. The MIDIS process has been introduced by several earlier publications $[8,9]$. Here, a description on that is presented in a stepwise fashion along with its key features and benefits. Also brief explanations are made upon key fabrication techniques of thin-film deposition and etching used in the process.

The MIDIS process is a high aspect-ratio bulk-micromachining process using three Single Crystal Silicon (SCS) wafers to be progressively assembled to form a final wafer stack. This standardized wafer-level packaging method provides benefits of small device footage, low product cost and reduced time for marketing and development [8]. Basically, the entire process can be divided into 13 steps. Figure 2 illustrates a generic MIDIS process flow.

The process starts with a $20-\mu$ m-deep bottom cavity to be formed on a handle wafer by using Deep Reactive-Ion Etching method (DRIE) to allow free vibrational motion of the resonator. This is processed by transferring the pattern on the photoresist deep down into the underlying silicon wafer through a combination of chemical and physical etching in a chamber filled with an inert gas [10]. The chemical process serves the primary function of etching silicon material through chemical reaction, while physical process helps remove passivation layer which 
inhibits the etching from continuing. Then, thermal oxidation is implemented in the second step to deposit a conformal layer of silicon dioxide thin film atop the handle wafer for electrical isolation purpose. In thermal oxidation process, high temperature $\left(900^{\circ} \mathrm{C}\right.$ and above) is applied to a quartz tube where the wafers are placed, which facilitates silicon wafers to react with oxygen atoms to form a layer of high-quality silicon dioxide, usually less than $1.5 \mu$ m thick. In MIDIS process, thickness of $1 \mu \mathrm{m}$ is required for silicon dioxide layer deposition.

The next step is to perform fusion bonding of two wafers, the processed handle wafer and a $30 \mu \mathrm{m}$-thick membrane wafer. Typical fusion bonding process involves mating of two wafers at room temperature and annealing process thereafter at a bonding temperature of over $1000^{\circ} \mathrm{C}$ [11]. In MIDIS process, bonding temperature is $1100^{\circ} \mathrm{C}$ and fusion bonding is processed under a high vacuum of 10 mtorr. This high vacuum-encapsulation level allows a resonator to achieve a $Q$ exceeding 20,000 [8]. The fourth step is to micromachine the membrane wafer using DRIE etching method to define the tuning fork resonator structure and electrodes in our design. The minimum feature size allowed on the device layer in MIDIS process is $1.5 \mu \mathrm{m}$ considering a device layer thickness of $30 \mu \mathrm{m}$, where the aspect ratio is the limit of DRIE process.

A third SCS interconnect wafer is used in the step that follows. It is etched by $20 \mu \mathrm{m}$ above the bottom surface through the same DRIE technique to form a top cavity. Later, based on completion of the previous step, another $2 \mu \mathrm{m}$ thickness is etched from deep bottom of the wafer using anisotropic wet etching method to form two inclined side walls (plane $\{111\}$ ) at an angle of $45.74^{\circ}$ to the device surface (plane $\{100\}$ ). This step serves a function of patterning the generic bonding plane for fusion bonding the interconnect wafer with the wafer assembly. Typical anisotropic etching of silicon is processed at an etching temperature of $80^{\circ} \mathrm{C}$ when the silicon wafer is drown in a chemical liquid compound of mixing Potassium Hydroxide (KOH) with water $\left(\mathrm{H}_{2} \mathrm{O}\right)$ at a ratio of 1 to 2 . For anisotropic wet etching process, the average etching rate of 


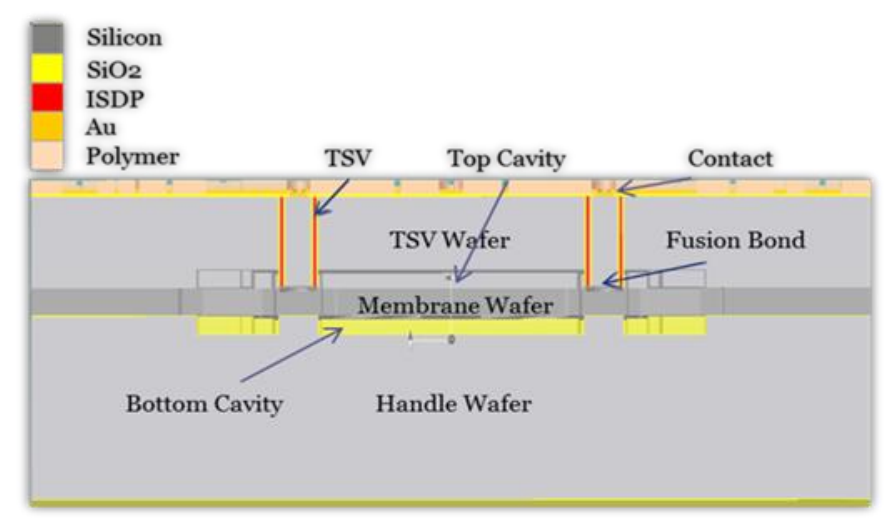

Figure 1. Cross-Sectional View of the 3D Resonator in MIDIS Process

a generic silicon wafer in a plane perpendicular to a direction vector of $\langle 100\rangle$ is about $1.4 \mu \mathrm{m} / \min [10]$.

The seventh step is taken to form Through Silicon Via (TSV) structures on the interconnect wafer. To complete this step, an isolated trench is made through the wafer from the top surface of each electrode by using DRIE etching technique and then filled with In-Situ Doped Polysilicon (ISDP) and silicon dioxide material. The purpose is to provide isolated electrical conductive pathways from contacts to where electrodes of different functions (eg. bias, actuation or sensing) can be given to the device layer.

Another layer of $1 \mu \mathrm{m}$-thick silicon dioxide is grown directly on TSV wafer in the eighth step, which aids to remove potential crosstalk between electrical paths on the surface of the TSV wafer. The thin-film deposition technique used in this step is Plasma Enhanced Chemical Vapor Deposition (PECVD). The process involves chemical reaction between two reactants $(\mathrm{Si}(\mathrm{OC} 2 \mathrm{H} 5) 4$ and $\mathrm{H} 2 \mathrm{O})$ in gas phase catalyzed by ion energy of plasma in a high pressured chamber where the silicon wafer is placed. The reaction products of the process are in solid phase and will be absorbed by and diffused into the surface of the wafer to form a layer of thin film [10]. The ninth step is to use Reactive lon Etching method to create space on the oxide 


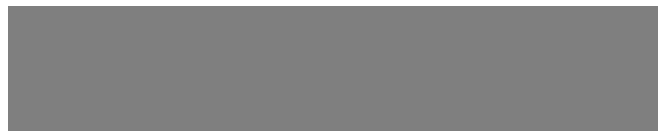

1. Handle Wafer

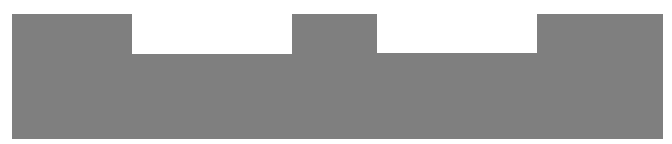

2. Etch bottom cavity

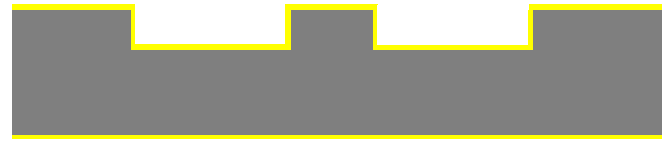

3. Deposit silicon dioxide

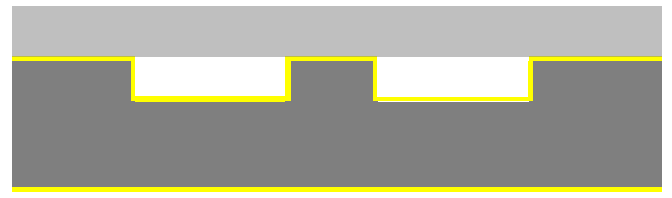

4. Stack Membrane Wafer

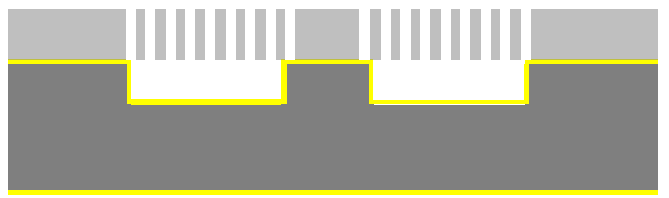

5. Pattern device structure

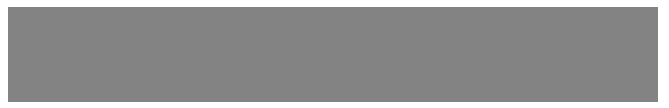

6. Interconnect Wafer

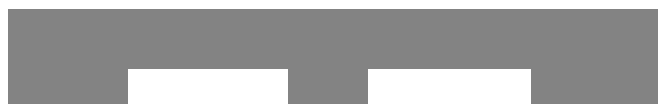

7. Etch top cavity

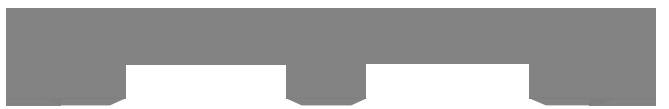

8. Etch bonding plane

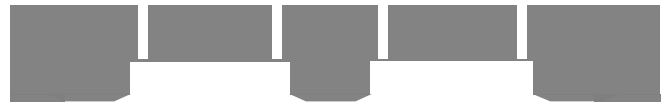

9. Etch trenches for TSV

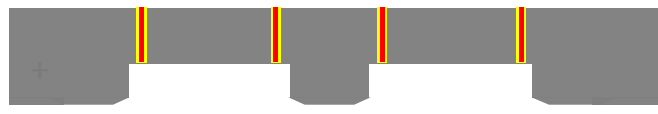

10. Fill materials in TSV trenches

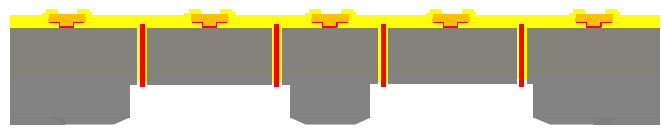

11. Deposit and etch $\mathrm{SO}_{2}$ and contact layer

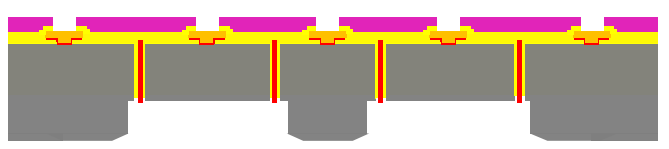

12. Deposit and etch passivation Layer

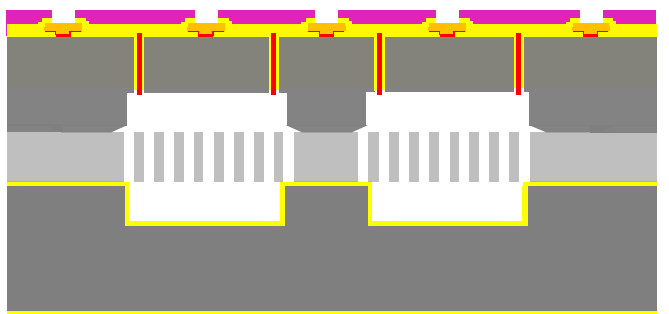

13. Fusion bond to form a wafer stack

Figure 2. MIDIS Process Flow Diagram

layer for extending the electrical conduction pathways towards the layer above, the contact layer.

The next step is to deposit a contact layer of $2 \mu \mathrm{m}$ thickness using Plasma Sputtering technique. In Plasma Sputtering process, thin film will be layered on the silicon wafer in a vacuum chamber, when ions of plasma generated in an inert gas (eg. Argon) bombard target material and force atoms of that material to be ejected and deposit on the wafer surface nearby 
[10]. The eleventh step is to pattern contacts, wires and bond pads on the contact layer by using RIE etching method. Then a $5 \mu$ m-thick passivation layer using Polymer material is grown as protection against corrosion atop the silicon dioxide layer. The MIDIS process ends when the processed interconnect wafer is finally fusion bonded with the device wafer at high temperature to remove any remnant gas or particles. 


\section{CHAPTER III}

\section{TUNING FORK RESONATOR DESIGN}

A 3D solid model of the $32 \mathrm{kHz}$ tuning fork resonator design used in this work is shown in Figure 3. The entire resonator device is designed to have a 3D size of $810 \mu \mathrm{m}$ (L) $\times 540 \mu \mathrm{m}(\mathrm{W}) \times 30 \mu \mathrm{m}(\mathrm{H})$. Its surface lies in a plane whose normal direction vector is aligned with crystal orientation of anisotropic material, silicon $<100>$. In our design, an electrostatic comb drive pair is used for device actuation and signal sensing through capacitive transduction. There are 147 interdigitated fingers, each $24 \mu \mathrm{m}$ long configured in both comb drives for the purpose of improving electrostatic coupling between the resonant part and electrodes. The resonant part, also named as proof mass, is suspended in vacuum over the bottom cavity through four fixed guided folded beam springs, placed symmetrical about the center point of the entire device. At the fixed ends to which the four springs extend, there are two anchors that measure $90 \mu \mathrm{m} \times 93 \mu \mathrm{m}$ in area. The conception of our tuning fork resonator design is derived from a comb-drive resonator design described in Reference [12]. Compared with the original design, our resonator has a unique characteristic that sensing and actuating electrodes are designed to be movable at $\mathrm{Y}$ axis to facilitate the transduction gap reduction process as a post fabrication step. This can be accomplished by providing the electrodes with serpentine spring supports for free vertical

movement as well as solid beam side supports to prohibit lateral movement. The use of those supports enables reduction of transduction gaps between the interweaving fingers and in turn meets the need of ultra-low DC polarization voltage and small motional 


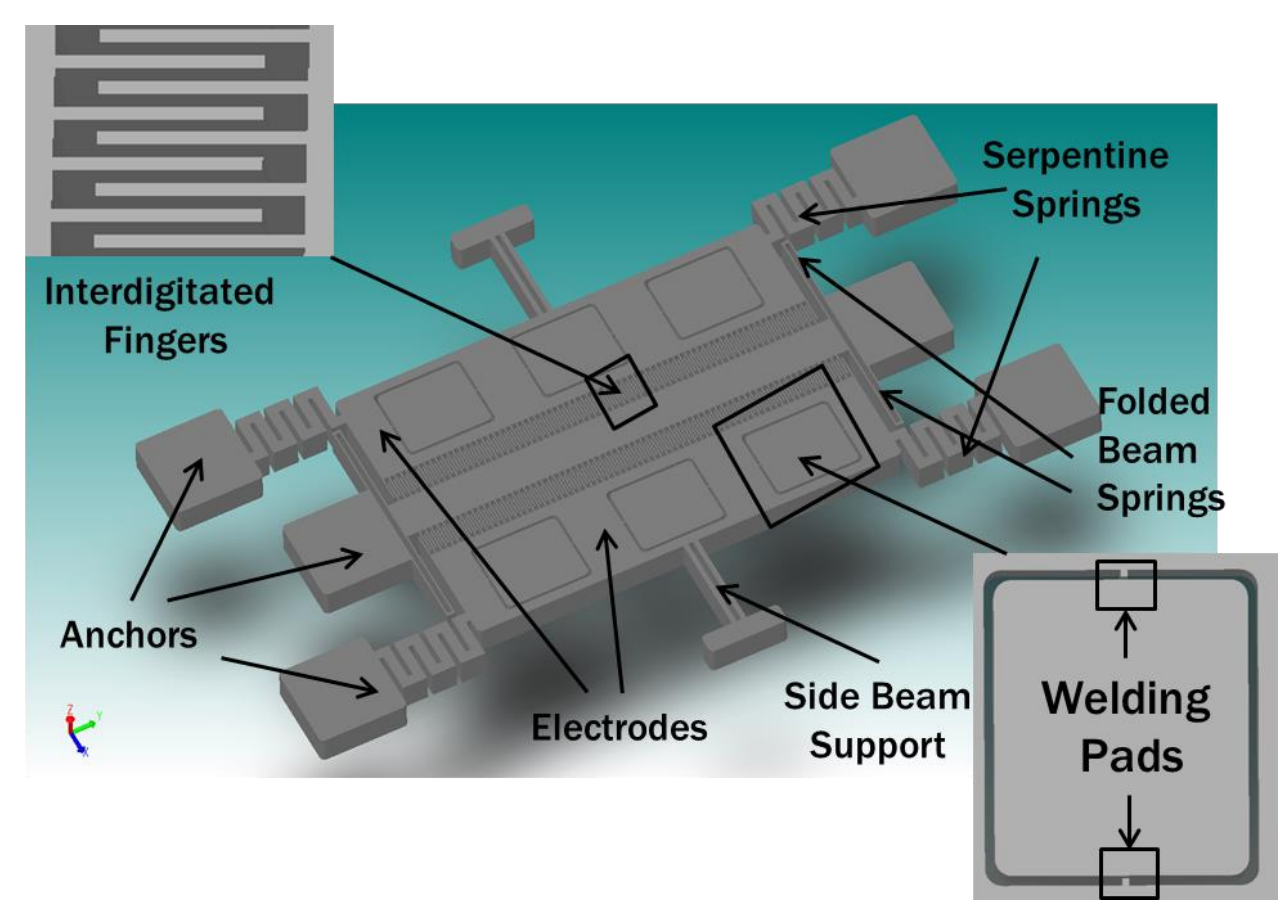

Figure 3. Tuning Fork Resonator Device Structure

resistance for the resonator. The transduction gap reduction methodology is discussed in detail next.

\subsection{Transduction Gap Reduction Technology}

According to TDSI MIDIS platform design rule guidelines, the minimum width of transduction gaps allowed is $1.5 \mu \mathrm{m}$ corresponding to $30 \mu \mathrm{m}$ membrane wafer thickness. This limitation is imposed mainly by high aspect ratio defined in DRIE processing for MIDIS process bulk micromaching, which is a ratio of 1 to 20. However, electrostatic MEMS resonators are in need of much smaller gap if they are designed to be commercially applicable. A gap of sub-micron scale may reduce the motional resistance of the resonator from hundreds or thousands of kilo Ohms to just several kilo Ohms, which greatly lowers the overall power consumption as well as improves the devices' power handling ability and phase noise control. Besides, the devices using MIDIS process are capable of operating 


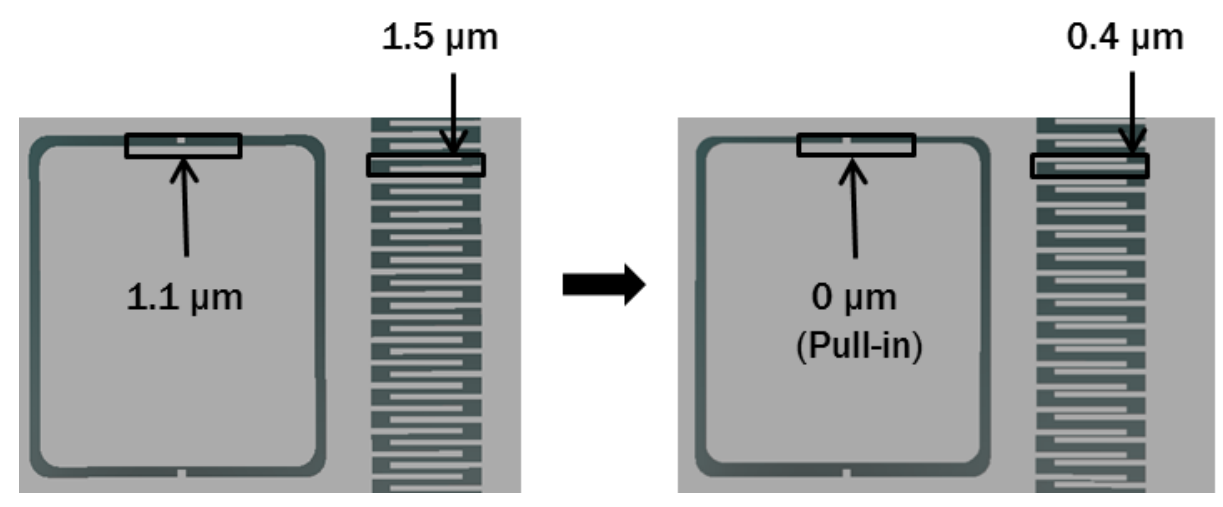

Figure 4. Transduction Gap Reduction Methodology

normally at a much lower DC polarization voltage if a small transduction gap size is possible. A novel post-fabrication gap reduction technique is presented herein to help relax minimum transduction gap restriction to as low as $50 \mathrm{~nm}$.

The process starts right after the resonator device is received from the foundry after fabrication[7]. A high DC voltage of about 50V is applied to generate electrical potential difference between movable electrodes and strategically located stop anchors enclosed by the electrodes. The induced electrostatic force will not stop pulling the electrodes towards the stop anchors until contact is made between them. Transduction gap width between adjacent interweaving fingers can now be reduced by the initial distance between each electrode and the stop anchors inside. To form a permanent connection for finalizing a new gap, a $10 \mathrm{~mA}$ short current pulse discharged by a capacitor is then used to melt the joints between welding-pads and movable electrodes. The two steps of reducing the transduction gap width by $1.1 \mu \mathrm{m}$ are illustrated in the flow chart Figure 4.

\subsection{Resonator Operation Theory}

Capacitive transduction, as an electromechanical operating mechanism, is used for this tuning fork resonator. A DC polarization voltage $\left(\mathrm{V}_{\mathrm{p}}\right)$ and an ultra-small AC voltage $\left(v_{i}\right)$ are 


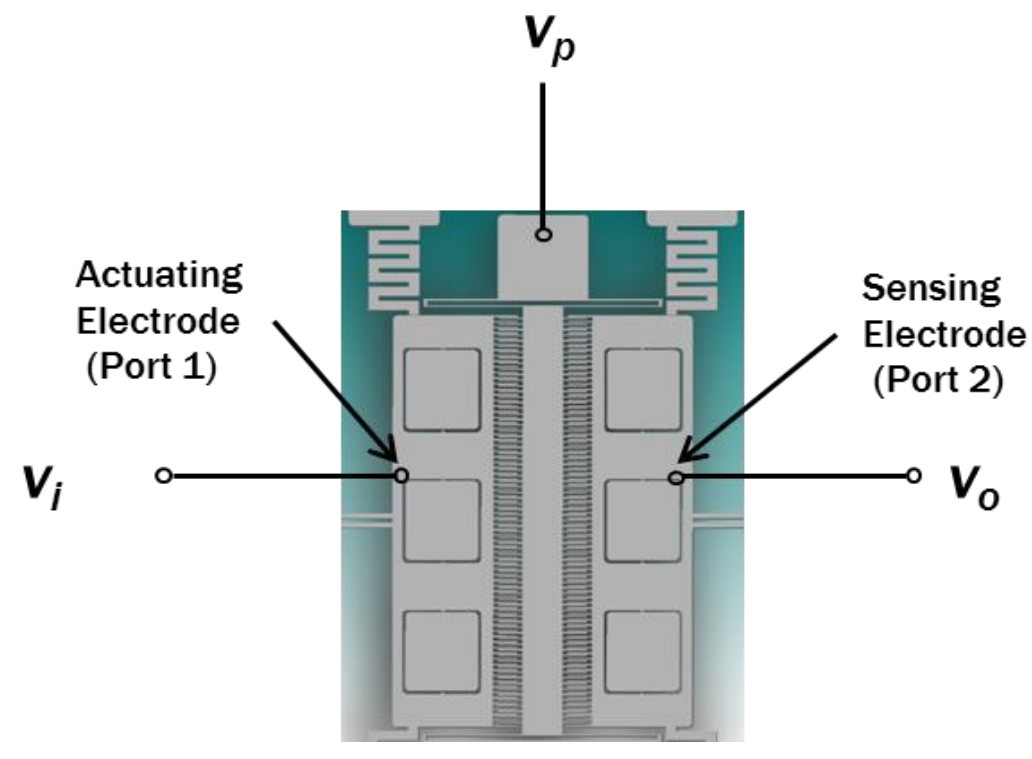

Figure 5. Two-Port Configuration

applied to the anchors of the movable structure and the actuating electrode respectively to activate the gap-reduced resonator device with a two-port configuration, shown in Figure 5. This produces an electrostatic force on the shuttle fingers (vibrating like shuttle buses) in a direction pointing towards the nearer electrode fingers, as the force exerted by those fingers is greater than that exerted by the electrode fingers further away in distance. A function of input electrostatic force $F_{i}$ expressed by $V_{p}$ and $v_{i}$ is then given below by Equation (1) [13],

$$
\begin{aligned}
F_{i}(t) & =\left|\frac{\partial w}{\partial x}\right|=\frac{1}{2}\left(\frac{\partial C_{1}}{\partial x}\right) V(t) \\
& =\frac{1}{2}\left(\frac{\partial C_{1}}{\partial x}\right)\left(V_{p}-v_{i}\right)^{2} \\
& =\frac{1}{2}\left(\frac{\partial C_{1}}{\partial x}\right)\left(V_{p}^{2}-2 V_{p} v_{i}+v_{i}^{2}\right) \\
& \approx-V_{p} v_{i}\left(\frac{\partial C_{1}}{\partial x}\right)
\end{aligned}
$$


where $V_{p} \gg v_{i}$, so only the dominant AC signal at resonance frequency is left when $V_{p}{ }^{2}$ is a DC term and $v_{i}^{2}$ is an ultra-weak signal and where $x$ is the displacement of the shuttle mass and $\frac{\partial C_{1}}{\partial x}$ is the change rate of the overlap capacitance between shuttle and electrode fingers at port 1 over the resonator vibrating distance. An expression of $\frac{\partial C_{1}}{\partial x}$ for port 1 is given by Equation (2)[14],

$$
C_{1}(x)=\frac{N \epsilon A}{g_{0}-x}=N C_{0}\left(1-\frac{x}{g_{0}}\right)^{-1} \rightarrow \frac{\partial C_{1}(x)}{\partial x}=\frac{N C_{0}}{g_{0}}\left(1-\frac{x}{g_{0}}\right)^{-2},
$$

where $\epsilon$ is the dielectric constant of free space, $g_{0}$ is the initial transduction gap after the gap reduction technique is applied, $\mathrm{C}_{0}$ and $\mathrm{A}$ are the static capacitance and the overlapping area for each electrode and shuttle finger pair, and $\mathrm{N}$ is the number of comb fingers at input side. Then the expression of $\partial C_{1} / \partial x$ can be expanded as Equation (3),

$$
\frac{\partial C_{1}}{\partial x}=\frac{N C_{0}}{g_{0}}\left(1+\frac{2 x}{g_{0}}+\frac{3 x^{2}}{g_{0}{ }^{2}}+\frac{4 x^{3}}{g_{0}{ }^{3}}+\cdots\right),
$$

If the displacement $x$ is insignificant compared with the static gap $g_{0}$, then all terms which contain $x$ in the equation above can be neglected as given by Equation (4),

$$
\frac{\partial C_{1}}{\partial x}=\frac{N C_{0}}{g_{0}}=\frac{N \varepsilon A}{g_{0}^{2}},
$$

Now considering a damped mechanical spring plus mass system, the equation of external force $F_{i}$ as a function of displacement $x$ is given by Equation (5),

$$
F_{i}=m_{e} \ddot{x}+c_{e} \dot{x}+k_{r e} x,
$$

where $k_{r e}$ is the total effective stiffness of the shuttle mass if the effect of electrical stiffness is considered, $c_{e}$ is the effective damping coefficient and $m_{e}$ is the effective mass. The full phasor form of equation (5) by Equation (6) [15], 


$$
\begin{gathered}
F_{i}(j w)=-w^{2} m_{e} x+k_{r e} x+c_{e} j w x \\
\downarrow \\
\frac{x}{F_{i}}(j w)=\left(\frac{1}{k_{r e}}\right)\left(-w^{2} \frac{m_{e}}{k_{r e}}+1+j w \frac{c_{e}}{k_{r e}}\right)^{-1},
\end{gathered}
$$

Also for a generic spring-mass system vibrating with harmonic motion, the resonance angular frequency and Quality factor at resonance can be calculated in Equations (7) and (8),

$$
\begin{aligned}
& \frac{k_{r e}}{m_{e}}=w_{0}{ }^{2}, \\
& Q=\frac{m_{e} w_{0}}{c_{e}}
\end{aligned}
$$

Inserting Equations (7) and (8) into Equation (6) results in a new expression of displacement over input force in phasor form as given by Equation (9),

$$
\frac{x}{F_{i}}(j w)=\frac{k_{r e}{ }^{-1}}{1-\left(\frac{w}{w_{0}}\right)^{2}+\frac{j w}{Q w_{0}}}
$$

Thus, for our MEMS device operating at resonance frequency, the ratio of displacement to input force $\frac{x}{F_{i}}$ can then be expressed as Equation (10),

$$
\frac{x}{F_{i}}=\frac{Q}{j k_{r e}}
$$

It is obvious from the equation above that the displacement $x$ is $90^{\circ}$ out of phase with respect to the input force $F_{i}$. When a DC bias voltage $V_{p}$ is applied, the time-varying capacitance $\partial C_{1} / \partial t$ combined with the bias voltage can source an output AC current $i_{o}$ at the sensing electrode, port 2. The current can be expressed by Equation (11),

$$
i_{o}=N V_{p} \frac{\partial C_{2}}{\partial t}
$$




$$
\begin{aligned}
& =N V_{p}\left(\frac{\partial C_{2}}{\partial x}\right)\left(\frac{\partial x}{\partial t}\right) \\
& =w_{0} N V_{p}\left(\frac{\partial C_{2}}{\partial x}\right)\left(\frac{\partial C_{1}}{\partial x}\right) \frac{Q V_{p}^{2} v_{i}}{k_{r e}} \\
& =w_{0} N V_{p}\left(\frac{N \varepsilon A}{g_{0}{ }^{2}}\right)^{2} \frac{Q V_{p}^{2} v_{i}}{k_{r e}}
\end{aligned}
$$

where Equations (1) and (10) are inserted in the function of $i_{o}$ and where $\partial C_{2} / \partial x$ is the overlapping capacitance between neighboring electrode and resonant fingers at port 2 . From the final form of the output current, we can conclude that $i_{o}$ is proportional to the amplitude of $v_{i}$ and oscillates at a same frequency of the input signal (frequency softening is not considered here).

Therefore, based on the above equations, the operating principle under the MEMSbased resonator device can be explained. Initially, when a DC bias voltage $V_{p}$ is applied to switch on the device, an ultra-small electrical input voltage $v_{i}$ is converted to a mechanical force $F_{i}$. It then induces a harmonic vibrating motion of the resonator at a frequency filtered by its inner mechanical property (spring stiffness and the total effective mass of the device). Through capacitive transduction, the displacement of the resonator is later converted back to a form of an electrical signal, as a harmonic current, $i_{o}$, at the output port.

To successfully actuate the device and sense a current signal with high resonance spectral purity, the DC polarization voltage applied to the device should be carefully selected. Theoretically, the voltage level cannot be too high to induce pull-in of the resonant fingers towards electrode fingers and should also be set high enough for output current of high spectral purity. Through simulations of the resonator design with different transduction gap sizes, a graph relating the gap size to its corresponding minimum DC bias voltage needed for the sensed current signal with high spectral purity is given by Figure 6. Figure 6 shows that the DC polarization voltage needed drops as the transduction gap size decreases, 


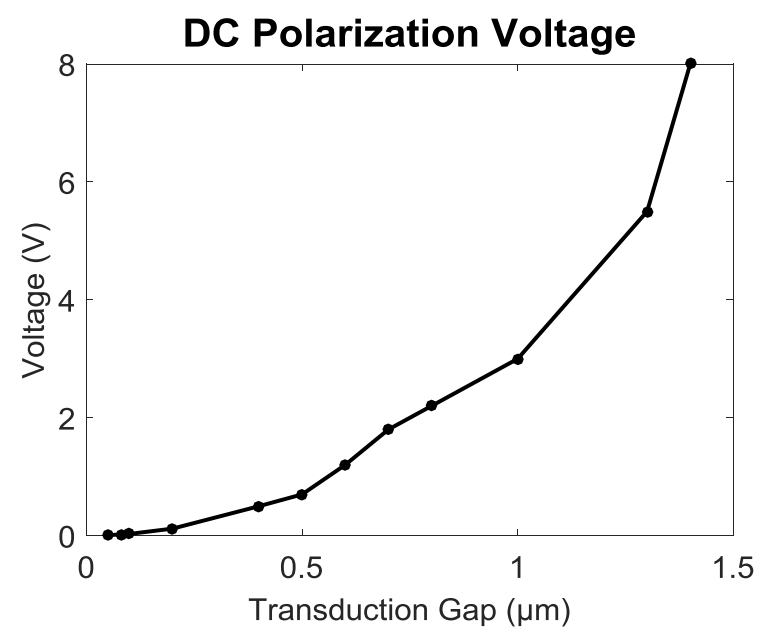

Figure 6. DC Bias Voltage over Frequency

which proves that using gap reduction methodology enables low DC bias voltage to drive the resonator device. The frequency response of harmonic current sensed from our resonator design using a $50 \mathrm{~nm}$ transduction gap size operating at $10 \mathrm{mV}$ is demonstrated as an example in Figure 7. In this graph, the peak value of the current gain is obtained at $32.7 \mathrm{kHz}$, which proves that the tuning fork resonator device with a transduction gap of 50nm is capable of operating normally when a DC polarization voltage of $10 \mathrm{mV}$ is applied.

\subsection{Resonance Frequency}

A simple resonance frequency expression of the tuning fork resonator in this work is given by Equation (12),

$$
f_{0}=\frac{1}{2 \pi}\left(\sqrt{\frac{k_{m}}{m_{e}}}\right),
$$

where $m_{e}$ is the effective mass of the resonator, $k_{m}$ is the mechanical stiffness of the folded beam spring structures. As seen from the Equation (12), the resonance frequency, $f_{0}$ is inversely related to the effective mass, $m_{e}$. Therefore, to achieve relatively low resonance frequency, about $32 \mathrm{kHz}$, a relatively large footprint of the device is an option. However, as the size is also a main factor for better electronic system integration, $k_{m}$ term has to be kept 


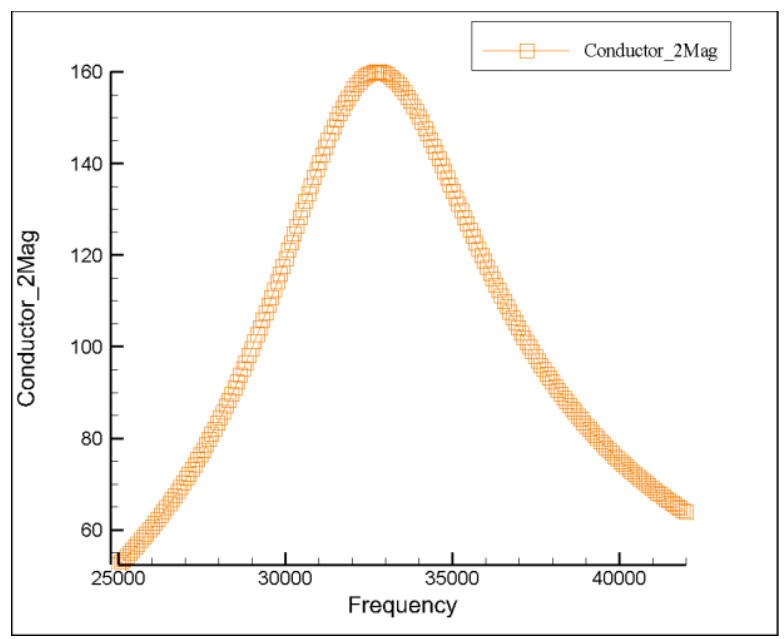

a. Harmonic Current Amplitude Response

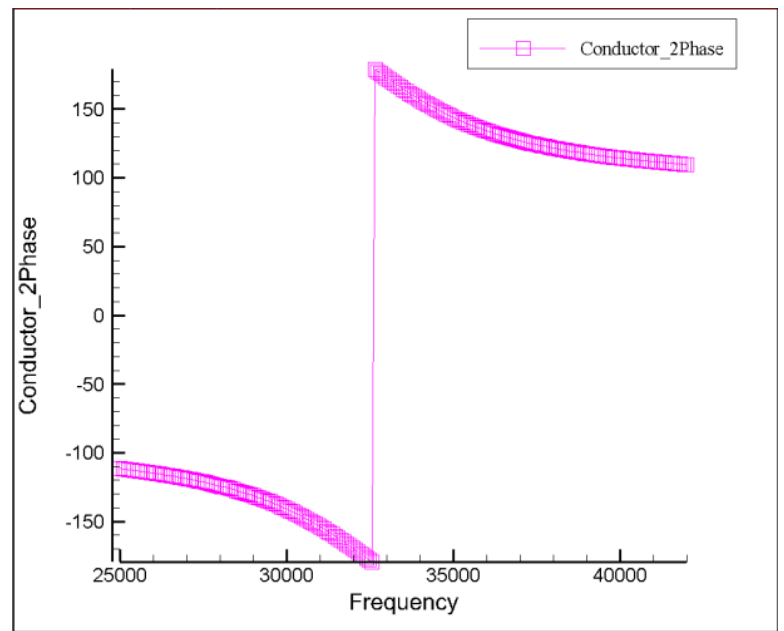

b. Harmonic Current Phase Response

Figure 7. Harmonic Current Frequency Response
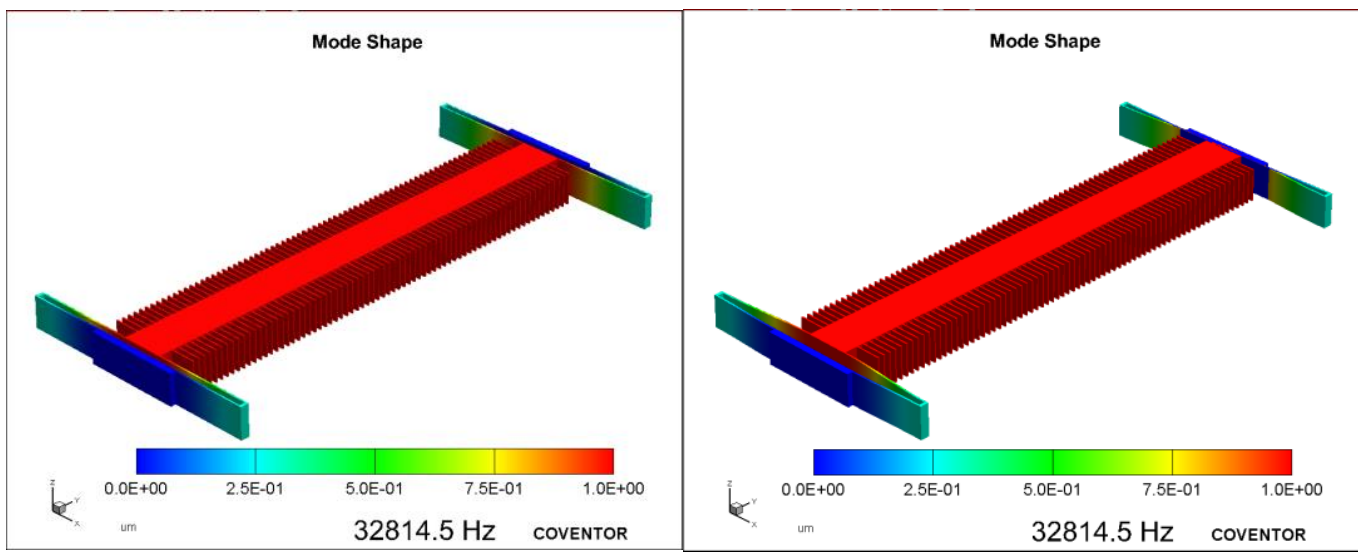

a. Mode Shape Starting Point

b. Mode Shape Ending Point

Figure 8. Resonator Mode Shape at $32.8 \mathrm{kHz}$ 
relatively small in order to balance a small $m_{e}$. As common knowledge regarding a solid beam structure or a double folded beam spring structure, using longer or thinner beams always result in less stiffness. That explains why the fixed guided folded beams for shuttle mass support in the current work are as designed, $142 \mu \mathrm{m}(\mathrm{L}) \times 1.6 \mu \mathrm{m}(\mathrm{W})$ in size.

Finite Element Modeling (FEM) through CoventorWare is used in the design process of the tuning fork resonator. Natural frequency of $32.81 \mathrm{kHz}$ is obtained for the transverse vibrating mode of the resonator in this work. The mode shape with exaggerated displacement for better visibility is demonstrated in Figure 8 , where different color indicates the gradient of modal displacement of the resonator.

\subsection{Frequency Softening Induced by Electrostatic Stiffness}

Though the input force expression can be finally reduced to a linear model when small signal terms are not considered, more components are actually generated for a complete description of the force. When the first two terms of Equation (3) is plugged into Equation (1), a new expression of the input force $F_{i}$ is given by Equation (13)[13],

$$
\begin{aligned}
& F_{i}(t)=\frac{1}{2}\left(\frac{\partial C_{1}}{\partial x}\right)\left(V_{p}^{2}-2 V_{p} v_{i}+v_{i}^{2}\right) \\
= & \frac{1}{2}\left(\frac{C_{0}}{g_{0}}\right)\left(1+\frac{2 x}{g_{0}}\right)\left(V_{p}^{2}-2 V_{p} v_{i}+v_{i}^{2}\right) \\
= & \frac{1}{2}\left(\frac{C_{0}}{g_{0}}\right)\left(V_{p}^{2}-2 V_{p} v_{i}+v_{i}^{2}+\frac{2 V_{p}^{2} x}{g_{0}}-\frac{4 V_{p} v_{i} x}{g_{0}}+\frac{2 v_{i}^{2} x}{g_{0}}\right) \\
\cong & \frac{1}{2}\left(\frac{C_{0}}{g_{0}}\right)\left(-2 V_{p} v_{i}+\frac{2 V_{p}^{2} x}{g_{0}}+\frac{2 v_{i}^{2} x}{g_{0}}\right),
\end{aligned}
$$

where only terms with components at resonance are retained in the final form. Considering an input voltage $v_{i}=V_{i} \cos _{0} t$ and a displacement expression of the resonator $x=X \operatorname{sinw}_{0} t$ with a $90^{\circ}$ phase shift, , the Equation (13) above can be further simplified as Equation (14) [13], 


$$
\begin{aligned}
F_{i}(t) & =\left(\frac{C_{0}}{g_{0}}\right)\left(-V_{p} V_{i} \cos w_{0} t+\frac{V_{p}^{2} X \sin w_{0} t}{g_{0}}+\frac{v_{i}^{2} X \sin w_{0} t}{g_{0}}\right) \\
& =-\left(\frac{C_{0}}{g_{0}}\right) V_{p} V_{i} \cos w_{0} t+\left(V_{p}^{2}+\left(V_{i} \cos w_{0} t\right)^{2}\right) \frac{C_{0}}{g_{0}^{2}} X \sin w_{0} t,
\end{aligned}
$$

where the first term is the input force component at resonance and the second term is proportional to the displacement amplitude, which serves as a contributor for the effective spring restoring force. However, instead of weakening the input force as a typical restoring force, this term acts to strengthen the external force and thus, undermines the mechanical spring stiffness, which induces spring softening phenomenon. Generally, this second term is defined as electrostatic stiffness as Equation (15),

$$
k_{e}=\left(V_{p}^{2}+\left(V_{i} \cos w_{0} t\right)^{2}\right) \frac{N \epsilon A}{g_{0}{ }^{3}}
$$

and the total effective stiffness can be modified Equation (16),

$$
k_{r e}=k_{m}-k_{e}
$$

and so the softened resonance frequency can then be expressed as Equation (17),

$$
\begin{aligned}
f_{r e} & =\frac{1}{2 \pi}\left(\sqrt{\frac{k_{m}-k_{e}}{m_{e}}}\right) \\
& =\frac{1}{2 \pi}\left(\sqrt{\frac{k_{r e}}{m_{e}}}\right),
\end{aligned}
$$

Through Finite Element Modeling (FEM) of the resonator device with different transduction gap sizes at minimum DC bias voltage for device excitation, a graph depicting the results of frequency softening effect analysis is shown in Figure 9. A conclusion can be drawn based on the graph that when the gap width reaches $50 \mathrm{~nm}$, the electrostatic stiffness is the weakest and the softened frequency can still stay at about $32.68 \mathrm{kHz}$. Thus, using a transduction gap of $50 \mathrm{~nm}$ for our resonator gives a best performance on lessoning the effect 


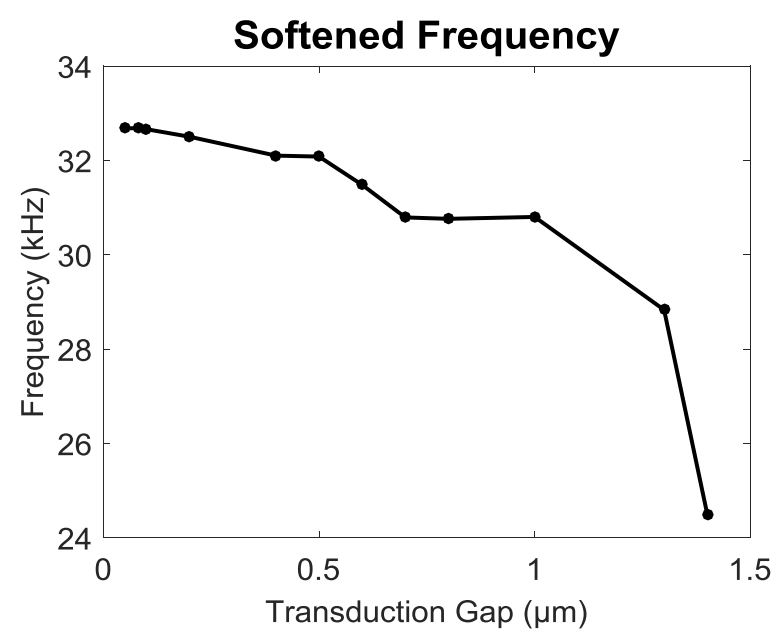

Figure 9. Softened Frequency over Transduction Gap Size

Table 1. Spring Softening Effect on The Resonator at $32.81 \mathrm{kHz}$

\begin{tabular}{|c|c|c|c|c|}
\hline & $\begin{array}{c}\text { Stressed } \\
\text { Frequency }\end{array}$ & $\begin{array}{c}\text { Generalized } \\
\text { Mass }\end{array}$ & $\begin{array}{c}\text { Electrostatic } \\
\text { Stiffness }\end{array}$ & $\begin{array}{c}\text { Softened } \\
\text { Frequency }\end{array}$ \\
\hline $\begin{array}{c}\text { Transverse } \\
\text { Mode }\end{array}$ & $3.28 \mathrm{E} 04$ & $1.68 \mathrm{E}-09$ & -0.6 & $3.268 \mathrm{E} 3$ \\
\hline
\end{tabular}

of frequency softening. Table 1 depicts the spring softening data of the resonator with the transduction gap of $50 \mathrm{~nm}$ and minimum DC bias voltage needed of just $10 \mathrm{mV}$. As seen from Table 1, the electrostatic stiffness is quite small and has little effect on the overall effective stiffness.

\subsection{Energy Loss Evaluation (Quality Factor)}

The Quality Factor (Q) plays a vital role in evaluating the performance of a MEMS-based resonator; that is, the higher the $Q$, the purer the electrical output signal spectrum will be at resonance frequency. It is a dimensionless parameter that is characterized by a ratio of resonator operating frequency to its minus $3 \mathrm{~dB}$ bandwidth. At operating frequency of an oscillation, higher $\mathrm{Q}$ indicates more energy stored relative to energy dissipated from the resonator and the oscillation dies out more slowly. And an expression of the loss mechanism affecting the $Q$ factor is described by Equation (18) [16], 


$$
\frac{1}{Q_{\text {total }}}=\frac{1}{Q_{T E D}}+\frac{1}{Q_{\text {Anchor }}}+\frac{1}{Q_{\text {Gas }}}+\frac{1}{Q_{\text {others }}}
$$

where $Q_{T E D}$ is a measurement of Thermal-Elastic Damping, $Q_{A n c h o r}$ is used to evaluate Anchor Dissipation, $Q_{A i r}$ is for Air Damping Test and $Q_{\text {Others }}$ covers all other energy loss mechanisms like thin structural loss and surface effect.

As the resonator design of this work can operate in an ultra-clean vacuum encapsulated environment through MIDIS platform, air damping owing to squeezed films or stokes is far less significant than other energy loss factors and is not measurable through Finite Element Modeling method. Likewise, structural loss or surface effect accounts for a very small proportion of the total Quality factor as well. As a result, only two contributors of the total Quality factor, Thermo-Elastic Damping and Anchor Loss, are evaluated by modeling the substrate, the resonator and electrodes in finite meshing size here.

\subsubsection{Thermo-Elastic Damping (TED) Analysis}

As is described in Reference [16], the Thermo-Elastic Damping (TED) is an intrinsic material damping source due to the irreversible energy transformation from the elastic field caused by deformation of the material to the thermal field. Strain of the body material due to vibrating motion induces temperature change such that compressive parts get hotter and tensile parts get cooler. As thermal energy generated cannot be converted back to elastic energy, heat conduction takes place all over the material and causes permanent energy dissipation, which in turn damps the vibrating motion of the solid body. A brief description on the process of analyzing the TED using FEM method is presented: First, when a DC voltage is applied, total electrostatic force exerted on all resonant fingers is measured through Finite Element Modeling using Coventorware. Then, the total pressure produced on overlapping areas between neighboring electrode and resonant fingers is given by Equation (19) (used later for TEM simulation), 


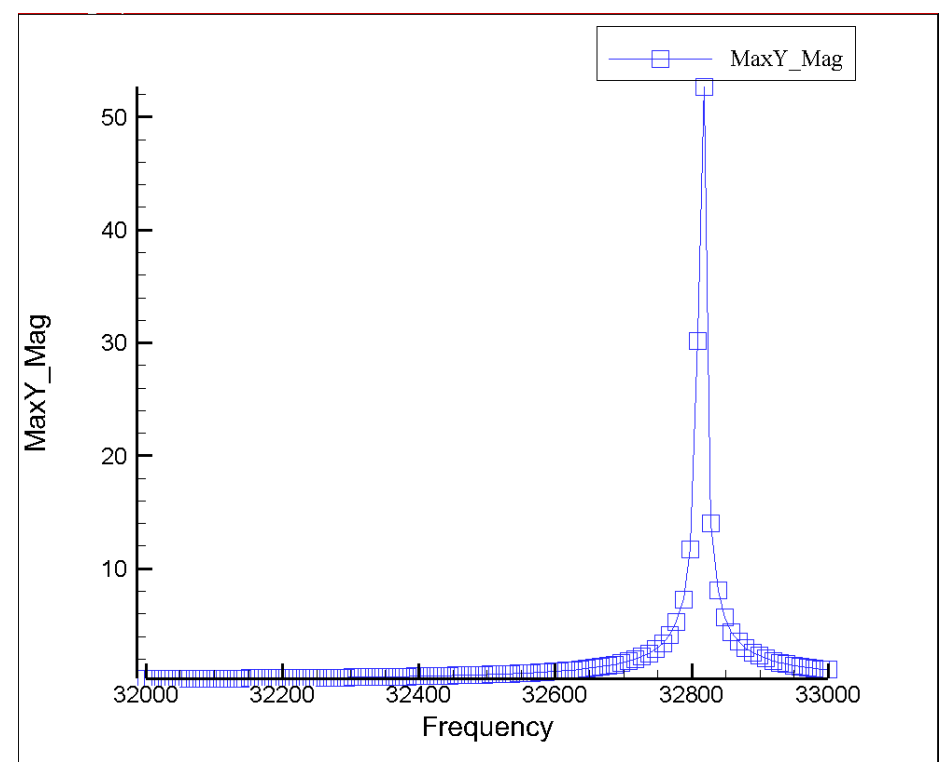

Figure 10. Y-axis Displacement over Frequency (TED)

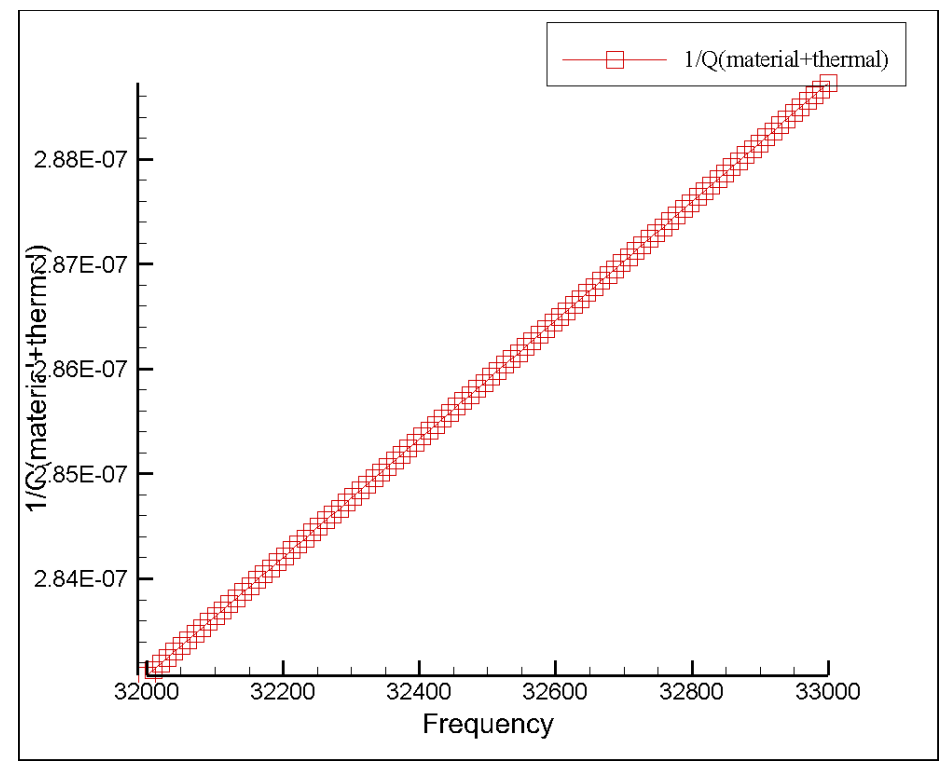

Figure 11. 1/ Q (TED) over Frequency

$$
P_{E}=\frac{F_{E}}{A_{o}}
$$

where $F_{E}$ is total electrostatic force generated on resonant fingers and $A_{O}$ is the overall overlapping area for capacitive coupling. The final step is to model the device excluding substrate and apply the result of $P_{E}$ on shuttle fingers as boundary condition for TED analysis. 
$1 / Q_{T E D}$ of about $2.88 \mathrm{e}-7$ is achieved from the simulation. Both graphs of $1 / Q_{T E D}$ and modal displacement of the resonator along $\mathrm{Y}$ axis over a frequency range between $32 \mathrm{kHz}$ and $33 \mathrm{kHz}$ are shown below in Figure 10 and 11 where Figure 10 indicates that a sharp displacement $(\mu \mathrm{m}$ unit) peak is detected at resonance frequency at which $Q_{T E D}$ is measured. And Figure 11 shows that $Q_{T E D}$ changes continuously and slowly over the specific frequency range.

\subsubsection{Anchor Loss Analysis and Overall Q Estimation}

Anchor Loss, often referred to as anchor damping, support loss, clamping loss or attachment loss, takes place when a portion of vibrating energy propagates into the structures to which the resonator device is linked. The energy that leaks through the anchors never returns and thus induces permanent energy dissipation[16]. For the resonator in this work, QuietBoundary used as a surface boundary condition is a solution in $Q_{\text {Anchor }}$ simulation. The working principle under it is that if a mechanical constraint is made to fix a solid model, the energy is reflected from the boundary surfaces and added back to its overall energy storage. Therefore, in our MEMS resonator design, QuietBoundary solution is used on the surfaces of the silicon substrate except the top surface where the anchor bottom interfaces to allow acoustic wave propagate beyond the boundary.

Through Finite Element Modeling and Quietboundary solution included, the final result of $1 / Q_{\text {Anchor }}$ turns out to be $1.34 \mathrm{e}-5$ at resonance. The graphs which illustrate the modal displacement along $\mathrm{Y}$ axis and Q's over a frequency range of $32 \mathrm{kHz}$ to $33 \mathrm{kHz}$ is presented in Figure 12 and Figure 13. Combining both graphs leads to a summarized result of $1 / Q_{\text {Anchor }}$. The total Quality factor $Q_{\text {total }}$ is then solved by inserting the results of $1 / Q_{T E D}$ and $1 / Q_{\text {Anchor }}$ to Equation (18) where the last two terms are not included for calculation. Thus, the final result of over 73,000 is attained for the total Quality Factor $Q_{\text {total }}$, which enables ultra-high spectral purity for a $32 \mathrm{kHz}$ clock signal for clocking application in low-frequency electronic systems. 


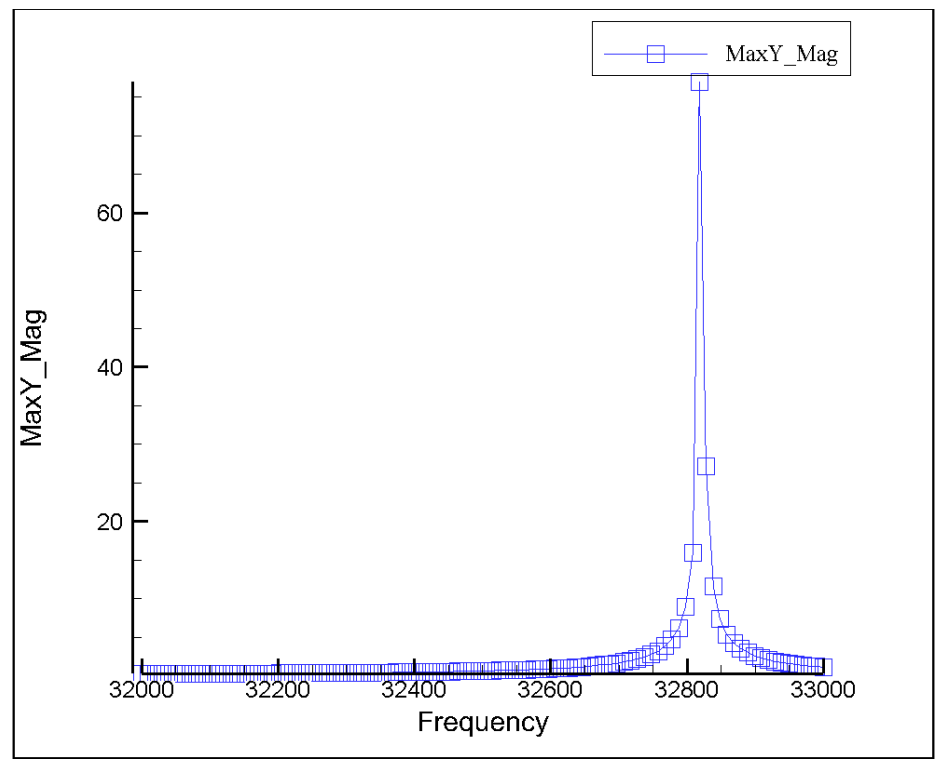

Figure 12. Y-axis Displacement Over Frequency (Anchor Loss)

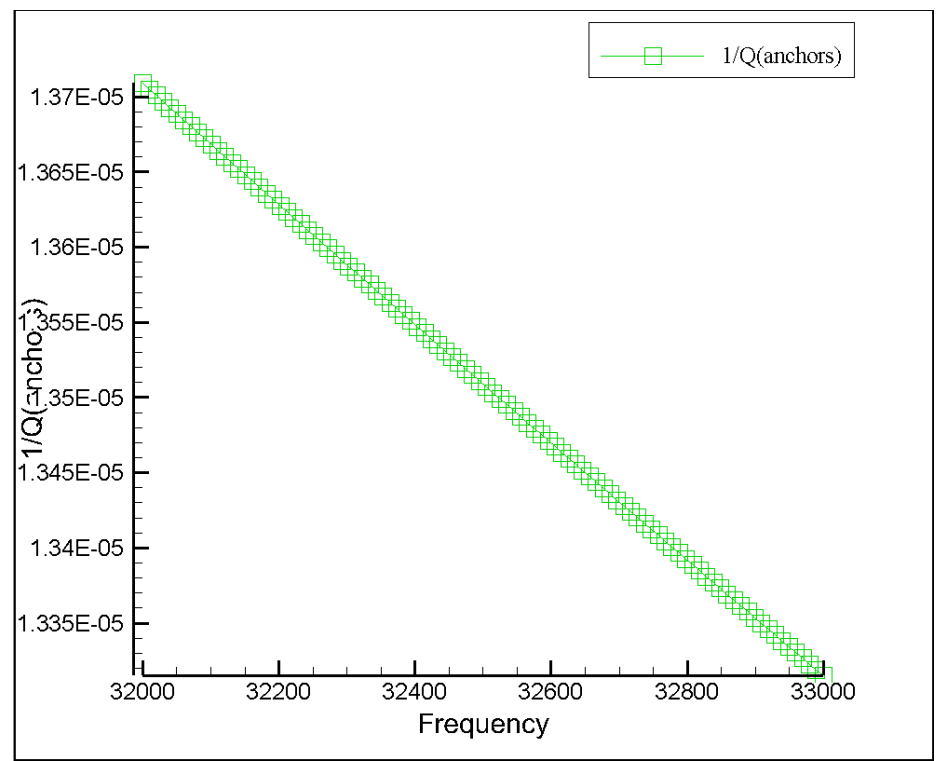

Figure 13. 1/ Q (Anchor Loss) over Frequency 


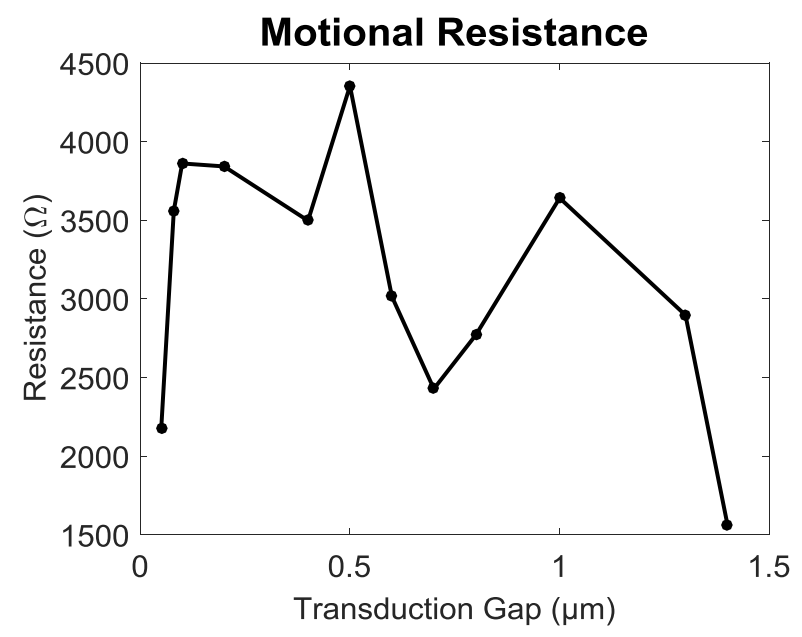

Figure 14. Motional Resistance vs. Transduction Gap Width

\subsection{Reduced Motional Resistance}

Motional resistance is another vital element at play when evaluating the performance of

a MEMS resonator. Low motional resistance of the micromechanical resonator not only helps to largely reduce the total power consumption of the MEMS-based oscillator, but helps improve its power handling ability. Generally, a relatively high motional resistance is nearly on the order of tens to hundreds $\mathrm{kHz}$. With the help of the transduction gap reduction technology, motional resistance can be minimized to an order of only several $\mathrm{kHz}$. By consulting the resonator design described in Reference [17] and the transverse comb drive concepts in Reference [14], the formulae for the motional resistance $R_{x}$ is given by Equation (20)

$$
R_{x}=\frac{\sqrt{k_{r e} \times m_{e}}}{Q \eta_{e 1} \eta_{e 2}},
$$

where $\eta_{e n}$ is the capacitive coupling factor between two neighboring electrode and resonant fingers at port $\mathrm{n}$. And $\eta_{\text {en }}$ can be calculated through the Equation (21) as,

$$
\eta_{e n}=N V_{p} \frac{\partial C_{n}}{\partial x}=N V_{p} \frac{\varepsilon t l}{g_{0}^{2}}
$$


where $\mathrm{N}$ is the number of comb fingers, $\mathrm{t}$ is the thickness of the device layer and $\mathrm{I}$ is the overlapping length between adjacent interactive fingers. Assuming $\eta_{e 1}=\eta_{e 2}=\eta$ and inserting Equations (17) and (21) into Equation (20), $R_{x}$ can be simplified to the expression as Equation (22) as,

$$
R_{x}=\frac{2 \pi f_{r e} g_{0}{ }^{4}}{Q N^{2} V_{p}^{2} \varepsilon^{2} t^{2} l^{2}}
$$

Based on the data from Figure 6 and Figure 9, a graph of motional resistance calculated for different transduction gap sizes is shown in Figure 14. A minimum resistance of about $2.2 \mathrm{k} \Omega$ is found at a gap of $50 \mathrm{~nm}$ (the spring softening effect is too strong at the gap of $1.4 \mu \mathrm{m}$ ), which means power consumption can be reduced by 10 to 100 times compared with other $32 \mathrm{kHz}$ MEMS-based resonator design $[5,6]$. 


\section{CHAPTER IV}

\section{CMOS SUSTAINING OSCILLATOR DESIGN}

The design of previously described tuning fork resonator is integrated with a sustaining amplifier to form an oscillator. Here, a transduction gap of $50 \mathrm{~nm}$ and a DC bias voltage of $10 \mathrm{mV}$ are selected as key characteristics of the resonator device used for oscillator configuration in terms of the performance on DC polarization voltage, electrostatic stiffness, power consumption and power handling (motional resistance). The integral components of the entire oscillator are presented in this chapter.

\subsection{Electrical Equivalent Circuit Model of the Resonator}

In order for the design and simulation of the MEMS-based oscillator, the mechanical form of the tuning fork resonator is converted to its corresponding electrical equivalent model for a two-port configuration, as is shown in Figure 15. The electrical circuit is formed by a series circuit of $L_{x}, C_{x}$ and $R_{x}$ connected with two $C_{p} \mathrm{~s}$ in parallel where $L_{x}, C_{x}$ and $R_{x}$ are motional inductance, capacitance and resistance and $C_{p}$ is the small parasitic capacitance between the electrodes and the substrate[6]. The results of $L_{x}, C_{x}$ are calculated in Equations (23) and (24) as,

$$
\begin{gathered}
L_{x}=\frac{m_{e}}{\eta^{2}}, \\
C_{x}=\frac{\eta^{2}}{k_{r e}},
\end{gathered}
$$




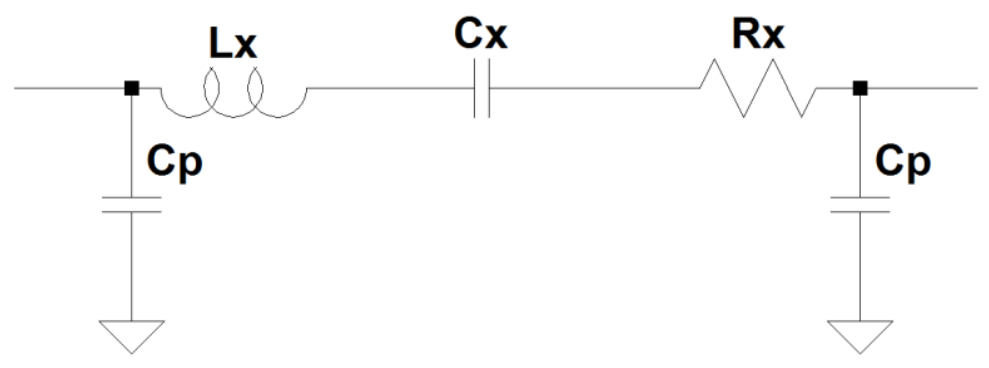

Figure 15. Electrical Equivalent Circuit Model

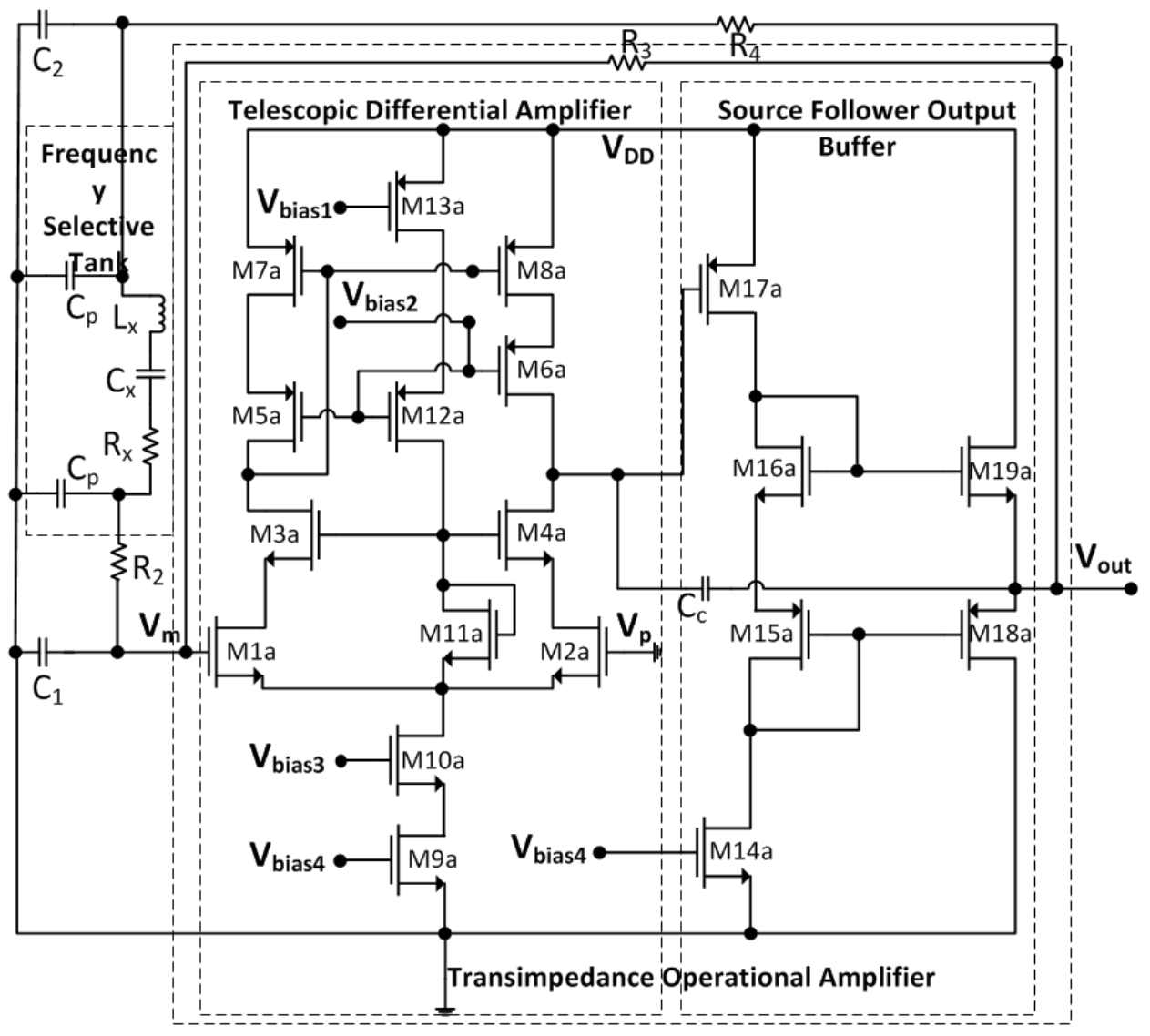

Figure 16. Overall Oscillator Circuit Topology

For the resonator with a transduction gap of $50 \mathrm{~nm}$ driven by a DC bias voltage of $10 \mathrm{mV}, L_{x}$, $C_{x}$ and $R_{x}$ are calculated as $772.61 \mathrm{H}, 30.7 \mathrm{fF}$ and $2.17 \mathrm{k} \Omega$ correspondingly by using Equations (23) and (24). Also the parasitic capacitance is assumed as $5 \mathrm{pF}$ for the worst case scenario. 


\subsection{CMOS Transimpedance Operational Amplifier}

In the oscillator circuitry, the sustaining amplifier serves as a form of CMOS transimpedance operational amplifier to compensate power dissipation through motional resistance of the resonator device. The operational amplifier, designed using Global Foundry (GF) $130 \mathrm{~nm}$ CMOS process, is a combination of an input-stage telescopic differential amplifier, an output-stage buffer using a source follower (common-drain amplifier) and a small capacitor in a compensation loop to stabilize circuit operation[18]. The circuit topology of the CMOS transimpedance amplifier is shown within the dash-line area in Figure 16. In addition, a compact physical layout design of the operational amplifier with biasing using the customized $130 \mathrm{~nm}$ CMOS process is presented in Figure 17.

The telescopic architecture of the input-stage differential amplifier provides a high gain, high output swing and low power consumption. The output stage is a class AB output buffer using a source follower configuration, which prevents a potential low resistance load from killing the gain and also lowers power consumption in this stage. In addition, a 100fF capacitor is connected between the outputs of both stages as direct compensation to stabilize the amplifier circuit. The amplifier circuit is voltage-biased by a constant- $\mathrm{g}_{\mathrm{m}}$ circuit as shown in Figure 18. In the oscillator circuitry, the sustaining amplifier serves as a form of CMOS transimpedance operational amplifier to compensate power dissipation through motional resistance of the resonator device. The parameters of all transistor devices can be found in Table 2. Simulations are conducted on the operational amplifier design using Silvaco EDA tools. Figure 19 shows the frequency response of the sustaining amplifier where a DC gain of about $53 \mathrm{~dB}$ is achieved, which is high enough to provide sufficient amplification of the sensed signal from the resonator device for normal operation of the oscillator. Besides, a bandwidth of about $300 \mathrm{kHz}$ is measured at the $-3 \mathrm{~dB}$ point, about ten times the target oscillating frequency. 


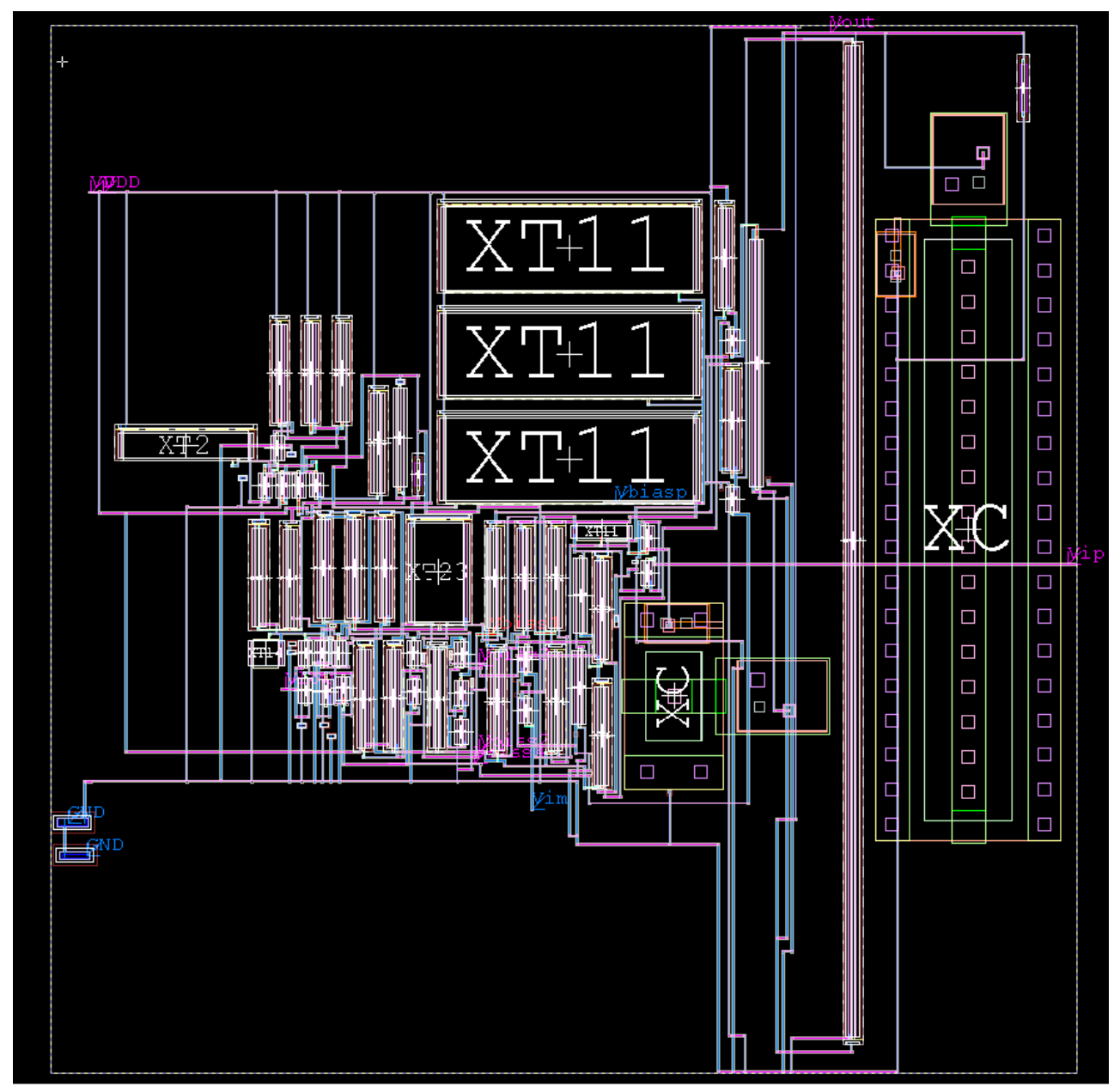

Figure 17. Physical Layout of the Operational Amplifier Circuit

This, as a condition plus a high phase margin of about $62^{\circ}$ measured at $0 \mathrm{~dB}$ frequency ensures high stability of the oscillation.

\subsection{CMOS-MEMS Oscillator}

Figure 16 demonstrates the overall CMOS-MEMS oscillator design where the sustaining CMOS transimpedance operational amplifier is connected in a positive feedback loop with the MEMS resonator electrical equivalent circuit model. The idea of the oscillator design in this work comes from the basic Colpitts oscillator configuration[19]. The operational principle under this configuration is utilizing a simple capacitive voltage divider as a source to continuously feed a 


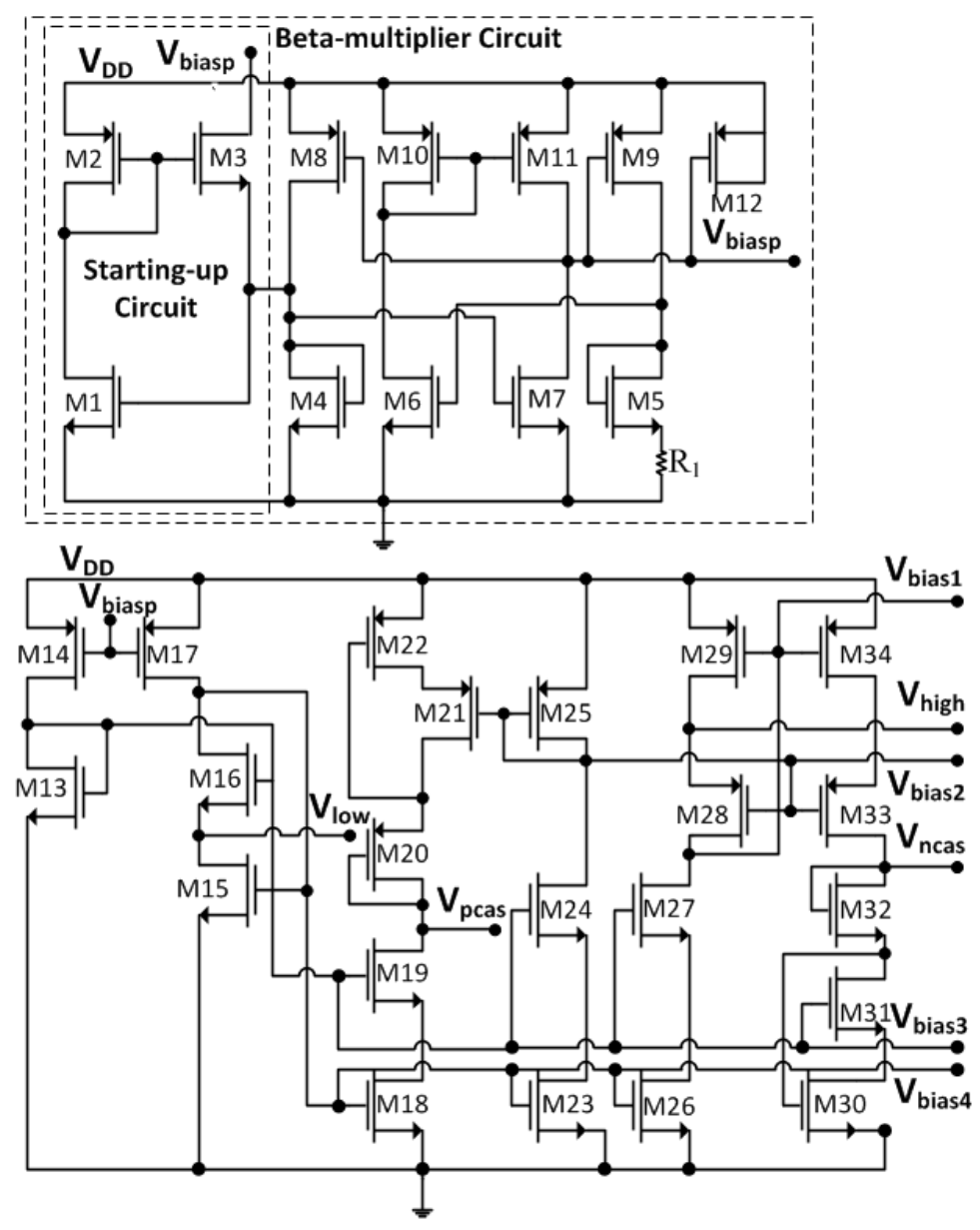

Figure 18. Biasing Circuit Schematic

proper fraction of the output voltage back to the input port until the total loop gain reaches an equilibrium of 1 . The amplitude of the oscillating electrical output signal then stops rising and reaches a steady value at operating frequency. Apart from the feedback system, a total loop phase shift of $360^{\circ}$ between the output and input port is satisfied as the oscillation condition [5]. This is achieved as a result of a $180^{\circ}$ phase shift of the transimpedance amplifier plus another $180^{\circ}$ phase shift contributed by the two load capacitors in the voltage divider system. The oscillating frequency of the sine-wave output signal is determined by the softened resonance frequency of the resonator, as a frequency setting element. In this work, the oscillating frequency 

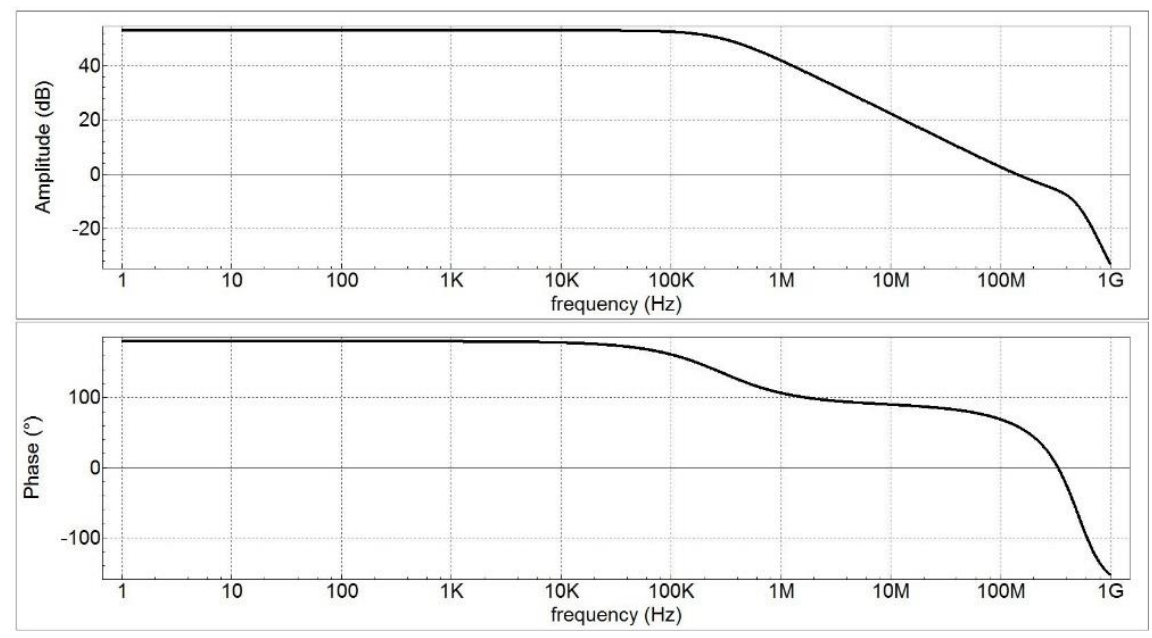

Figure 19. Frequency Response of the Operational Amplifier

Table 2. Transistor Parameters of The Transimpedance Operational Amplifier

\begin{tabular}{|c|c|c|c|}
\hline Device & Type & $\mathrm{W}(\mu \mathrm{m})$ & $\mathrm{L}(\mu \mathrm{m})$ \\
\hline $\begin{array}{c}\text { M1, M3, M4, M6-7, } \\
\text { M15-16, M18-19, } \\
\text { M23-24, M26-27, } \\
\begin{array}{c}\text { M30-32, M1a-4a, } \\
\text { M14a, M16a }\end{array}\end{array}$ & $\mathrm{N}$ & 2.4 & 0.24 \\
\hline $\begin{array}{c}\text { M5, M8-11, M14, } \\
\text { M17, M20-22, M28- } \\
\text { 29, M33-34, M5a-8a, } \\
\text { M12a-13a, M15a, } \\
\text { M17a }\end{array}$ & $\mathrm{P}$ & 9.6 & 0.24 \\
\hline $\mathrm{M} 13$ & $\mathrm{~N}$ & 2.4 & 2.4 \\
\hline $\mathrm{M} 9 \mathrm{a}-10 \mathrm{a}$ & $\mathrm{N}$ & 7.2 & 0.24 \\
\hline $\mathrm{M} 19 \mathrm{a}$ & $\mathrm{N}$ & 24 & 0.24 \\
\hline $\mathrm{M} 11 \mathrm{a}$ & $\mathrm{N}$ & 1.2 & 4.8 \\
\hline $\mathrm{M} 18 \mathrm{a}$ & $\mathrm{P}$ & 96 & 0.24 \\
\hline $\mathrm{M} 2$ & $\mathrm{P}$ & 2.4 & 12 \\
\hline $\mathrm{M} 12$ & $\mathrm{P}$ & 24 & 24 \\
\hline $\mathrm{M} 25$ & $\mathrm{P}$ & 9.6 & 4.8 \\
\hline
\end{tabular}

is about $32.7 \mathrm{kHz}$. The parameters of all resistors, capacitors and inductors used in the oscillator circuit are given in Table 3. 
Table 3. Parameters of All Resistors, Inductors and Capacitors Used

\begin{tabular}{|c|c|c|}
\hline Device & Value & Unit \\
\hline $\mathrm{R}_{1}$ & 5 & $\mathrm{k} \Omega$ \\
\hline $\mathrm{R}_{2}$ & 3080 & $\mathrm{k} \Omega$ \\
\hline $\mathrm{R}_{3}$ & 6000 & $\mathrm{k} \Omega$ \\
\hline $\mathrm{R}_{4}$ & 1500 & $\mathrm{k} \Omega$ \\
\hline $\mathrm{R}_{\mathrm{x}}$ & 4.24 & $\mathrm{k} \Omega$ \\
\hline $\mathrm{C}_{\mathrm{x}}$ & 50.53 & $\mathrm{fF}$ \\
\hline $\mathrm{C}_{\mathrm{p}}$ & 5 & $\mathrm{pF}$ \\
\hline $\mathrm{C}_{1}, \mathrm{C}_{2}$ & 1 & $\mathrm{pF}$ \\
\hline $\mathrm{C}_{\mathrm{c}}$ & 100 & $\mathrm{fF}$ \\
\hline $\mathrm{L}_{\mathrm{x}}$ & 480.43 & $\mathrm{H}$ \\
\hline
\end{tabular}

Table 4. DC Operating Point Analysis

\begin{tabular}{|l|l|l|}
\hline Parameter & DC Value & Unit \\
\hline V(VDD) & 1.2 & $\mathrm{~V}$ \\
\hline$I_{s}(\mathrm{M} 7 \mathrm{a})$ & 9.06 & $\mu \mathrm{A}$ \\
\hline $\mathrm{I}_{s}(\mathrm{M} 13 \mathrm{a})$ & 11.85 & $\mu \mathrm{A}$ \\
\hline $\mathrm{I}_{s}(\mathrm{M} 8 \mathrm{a})$ & 9.08 & $\mu \mathrm{A}$ \\
\hline $\mathrm{I}_{s}(\mathrm{M} 17 \mathrm{a})$ & 31.69 & $\mu \mathrm{A}$ \\
\hline $\mathrm{I}_{s}(\mathrm{M} 19 \mathrm{a})$ & 339.5 & $\mu \mathrm{A}$ \\
\hline $\mathrm{I}_{s}(\mathrm{M} 2)$ & 3.23 & $\mu \mathrm{A}$ \\
\hline $\mathrm{I}_{s}(\mathrm{M} 8)$ & 13.18 & $\mu \mathrm{A}$ \\
\hline $\mathrm{I}_{s}(\mathrm{M} 10)$ & 15.84 & $\mu \mathrm{A}$ \\
\hline $\mathrm{I}_{s}(\mathrm{M} 11)$ & 15.69 & $\mu \mathrm{A}$ \\
\hline $\mathrm{I}_{s}(\mathrm{M} 9)$ & 13.17 & $\mu \mathrm{A}$ \\
\hline $\mathrm{I}_{s}(\mathrm{M} 14)$ & 13.11 & $\mu \mathrm{A}$ \\
\hline $\mathrm{I}_{s}(\mathrm{M} 17)$ & 12.33 & $\mu \mathrm{A}$ \\
\hline $\mathrm{I}_{s}(\mathrm{M} 22)$ & 11.16 & $\mu \mathrm{A}$ \\
\hline $\mathrm{I}_{s}(\mathrm{M} 25)$ & 12.73 & $\mu \mathrm{A}$ \\
\hline $\mathrm{I}_{s}(\mathrm{M} 29)$ & 12.93 & $\mu \mathrm{A}$ \\
\hline $\mathrm{I}_{s}(\mathrm{M} 34)$ & 11.56 & $\mu \mathrm{A}$ \\
\hline $\mathrm{I}_{s s u m}$ & 536.11 & $\mu \mathrm{A}$ \\
\hline
\end{tabular}

Again, several analysis are done upon the entire oscillator circuit design, including the operating point evaluation, the frequency response of the oscillator ( $A C$ analysis) and the timedomain output signal analysis (transient analysis). The frequency response is demonstrated in Figure 20 where the peak amplitude lies at a frequency of around $32.7 \mathrm{kHz}$. This shows that the 

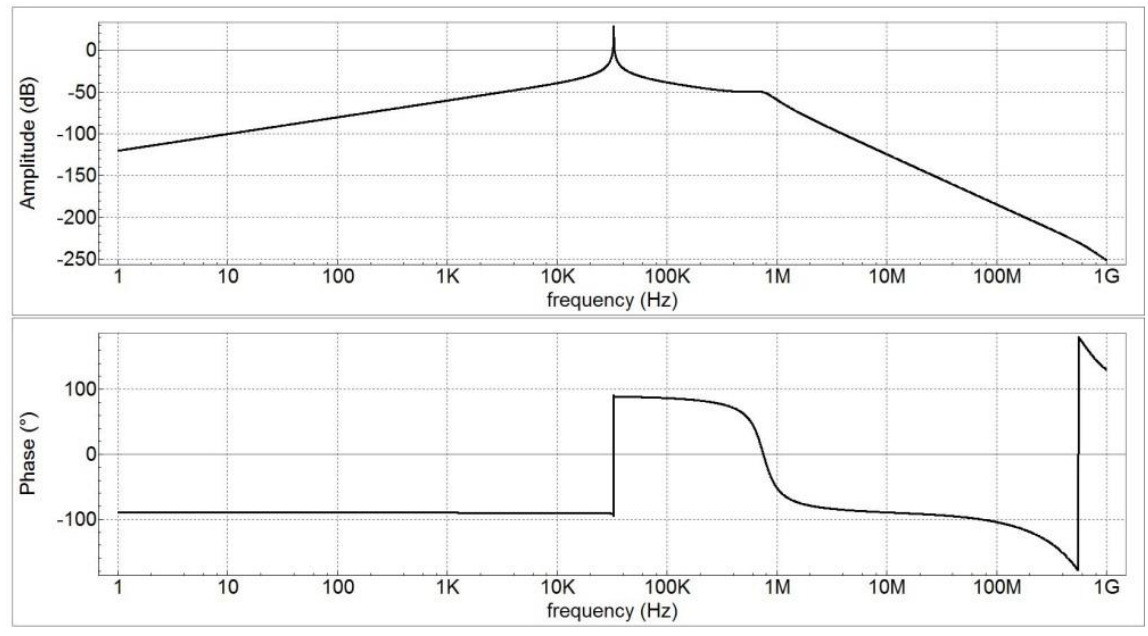

Figure 20. Frequency Response of the Oscillator

oscillator device, when operating normally, can generate a sine wave signal oscillating purely at that frequency. The sine wave signal is presented in Figure 21 with a voltage swing of about $92 \mathrm{mV}$ and DC shift at about $0.6 \mathrm{~V}$. Though the output swing is not distinctly high, as it is restricted by the low DC power supply (1.2V) used in circuit design, the performance of power consumption is quite acceptable. Total power consumption of the oscillator circuit can be calculated through the Equation (25) as,

$$
P_{t}=V_{D D} \times \mathrm{I}_{\text {Ssum }},
$$

Based on the results from Table 4 and Equation (25), the total power consumption $P_{t}$ turns out to be $643.33 \mu \mathrm{W}$. Figure 22 shows that the signal starts oscillating with a steady amplitude after $1.8 \mathrm{~s}$, which means stable oscillation is achieved over time for the oscillator design of this work. 


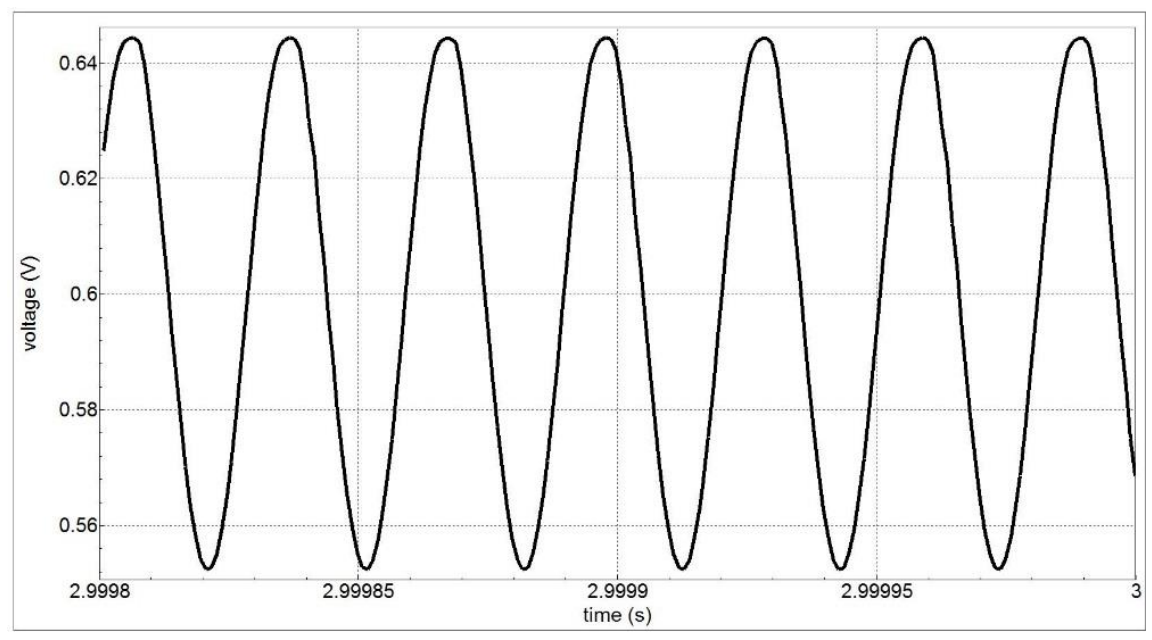

Figure 21. Output Signal in Time-domain

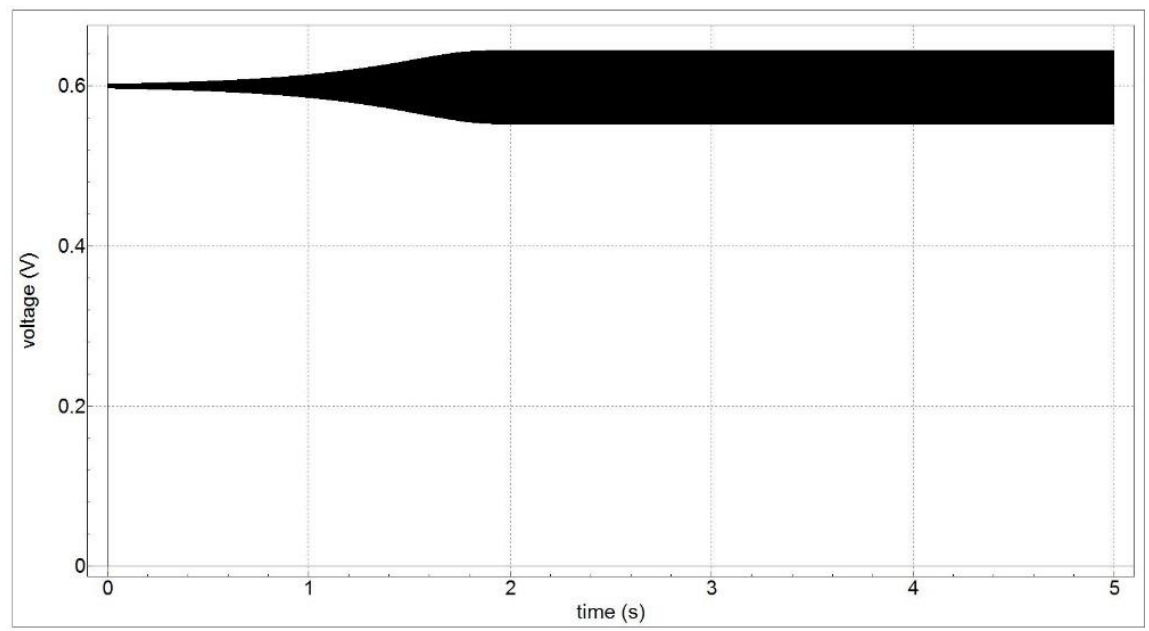

Figure 22. Output Signal over Time 


\section{CHAPTER V}

\section{CONCLUSION AND VISION OF FUTURE WORK}

\subsection{Conclusion}

A detailed analysis is performed in this thesis upon the design of $32 \mathrm{kHz}$ wafer-level vacuum-encapsulated silicon MEMS tuning fork resonator in terms of the effect of the gapreduction methodology along with its capability of being integrated with a CMOS circuit on a system level. The novel gap-reduction technology helps reduce DC polarization voltage to a sub$1 \mathrm{~V}$ level (as low as $10 \mathrm{mV}$ ) and lower the motional resistance of the resonator by 10 to 100 times, to about $2 \mathrm{k} \Omega$ as a minimum. In addition, the spring stiffness softening effect on the resonator device, commonly seen on transverse comb drive structures, is largely cut down using gap reduction technique, which minimizes the frequency loss incurred by a direct relation between the bias voltage and the electrostatic stiffness. A sustaining transimpedance amplifier designed using GF $130 \mathrm{~nm}$ CMOS process assists with a steady and continuous oscillation of the output electrical signal. Power consumption of the oscillator is controlled to around $600 \mu \mathrm{W}$.

\subsection{Vision of Future Work}

In this section, the prospect of subsequent work on this thesis project is briefly discussed. From previous description of the tuning fork resonator design, a small unexpected electrical stiffness still exists as the working mechanism of the resonator in this work is similar to that of a transverse comb drive structure, which causes a frequency difference between the 
natural resonance frequency and output signal frequency. Therefore, how transduction gap reduction can be applied to laterally moving comb drive structures has to be studied, as the frequency is completely irrelevant to the bias voltage and capacitance change across the electrodes and the shuttle fingers.

Then improvement on the sustaining operational amplifier design used in this work will be taken into account, as performance such as total power consumption and output voltage swing of the oscillator is primarily determined by the amplifier itself. An operational amplifier with better output swing and even lower power consumption at no expense of gain is an ideal choice.

In addition, time delay for an oscillator to start oscillating at a steady state is desired to be shortened. This probably can be realized by integrating the MEMS-based structure with the CMOS amplifier in another oscillator configuration or attempting to reduce all resistance and capacitance values used in the oscillator that determines time constant as a contributor for the total time delay of the overall oscillator circuit. 


\section{BIBLIOGRAPHY}

[1] C. T. Nguyen, "MEMS technology for timing and frequency control," IEEE Trans Ultrason Ferroelectr Freq Control, vol. 54, no. 2, pp. 251-70, Feb 2007.

[2] J. T. M. van Beek and R. Puers, "A review of MEMS oscillators for frequency reference and timing applications," (in English), Journal of Micromechanics and Microengineering, vol. 22, no. 1, Jan 2012.

[3] K. R. Cioffi and W. T. Hsu, "32KHz MEMS-based oscillator for low-power applications," (in English), 2005 IEEE International Frequency Control Symposium and Exhibition, pp. 551-558, 2005.

[4] D. E. Serrano, R. Tabrizian, and F. Ayazi, "Tunable Piezoelectric MEMS Resonators for Real-Time Clock," (in English), 2011 Joint Conference of the leee International Frequency Control Symposium/European Frequency and Time Forum Proceedings, pp. 765-768, 2011.

[5] H. G. Barrow et al., "A Real-Time 32.768-kHz Clock Oscillator Using a 0.0154-mm(2) Micromechanical Resonator Frequency-Setting Element," (in English), 2012 leee International Frequency Control Symposium (Fcs), 2012.

[6] S. Zaliasl et al., "A 3 ppm 1.5 x 0.8 mm(2) 1.0 mu A 32.768 kHz MEMS-Based Oscillator," (in English), leee Journal of Solid-State Circuits, vol. 50, no. 1, pp. 291-302, Jan 2015.

[7] M. Nowack et al., "Micro arc welding for electrode gap reduction of high aspect ratio microstructures," (in English), Sensors and Actuators a-Physical, vol. 188, pp. 495-502, Dec 2012.

[8] MIDIS: MEMS Integrated Design for Inertial Sensors [Online]. Available: http://www.teledynedalsa.com/semi/mems/applications/midis/

[9] G. Xereas and V. P. Chodavarapu, "Wafer-Level Vacuum-Encapsulated Lame Mode Resonator With $\mathrm{f}-\mathrm{Q}$ Product of $2.23 \times 10(13) \mathrm{Hz}$," (in English), leee Electron Device Letters, vol. 36, no. 10, pp. 1079-1081, Oct 2015.

[10] C. Liu, Foundations of MEMS, 2 ed. Upper saddle river: Prentice hall, 2012, p. 576 s. 
[11] C. Harendt, B. Hofflinger, H. G. Graf, and E. Penteker, "Silicon Direct Bonding for Sensor Applications - Characterization of the Bond Quality," (in English), Sensors and Actuators a-Physical, vol. 25, no. 1-3, pp. 87-92, Oct-Jan 1991.

[12] E. Carr, S. Olivier, and O. Solgaard, "Large-stroke self-aligned vertical comb drive actuators for adaptive optics applications," (in English), Mems/Moems Components and Their Applications lii, vol. 6113, 2006.

[13] W. Jing, Z. Y. Ren, and C. T. C. Nguyen, "1.156-GHz self-aligned vibrating micromechanical disk resonator," (in English), leee Transactions on Ultrasonics Ferroelectrics and Frequency Control, vol. 51, no. 12, pp. 1607-1628, Dec 2004.

[14] C. Liu, Foundations of MEMS, 2nd ed. Upper Saddle River, N.J.: Prentice Hall, 2012, pp. xiv, 562 p.

[15] G. T. A. Kovacs, Micromachined transducers sourcebook. Boston, Ma.: WCB, 1998, pp. $\mathrm{xx}, 911,3 \mathrm{p}$.

[16] MEMS resonator: Thermo-Elastic Damping and Anchor Loss models [Online]. Available: http://info.coventor.com/memsahead/bid/34328/MEMS-resonator-Thermo-ElasticDamping-and-Anchor-Loss-models

[17] C. T. C. Nguyen and R. T. Howe, "An integrated CMOS micromechanical resonator high-Q oscillator," (in English), leee Journal of Solid-State Circuits, vol. 34, no. 4, pp. 440-455, Apr 1999.

[18] R. J. Baker and Institute of Electrical and Electronics Engineers., CMOS circuit design, layout, and simulation, Rev. 2nd ed. (IEEE Press series on microelectronic systems). Piscataway, NJ Hoboken, NJ: IEEE Press ; Wiley-Interscience, 2008, pp. xxxi, 1038 p.

[19] C. Y. Chung and S. Y. Chao, "Robust Colpitts And Hartley Oscillator Design," (in English), 2014 leee International Frequency Control Symposium (Fcs), pp. 141-146, 2014. 


\section{APPENDIX A \\ HARMONIC CURRENT OF THE RESONATOR WITH DIFFERENT TRANSDUCTION GAP SIZES}

1. Gap Size: $1.4 \mu \mathrm{m}$ and DC Bias Voltage: $8 \mathrm{~V}$
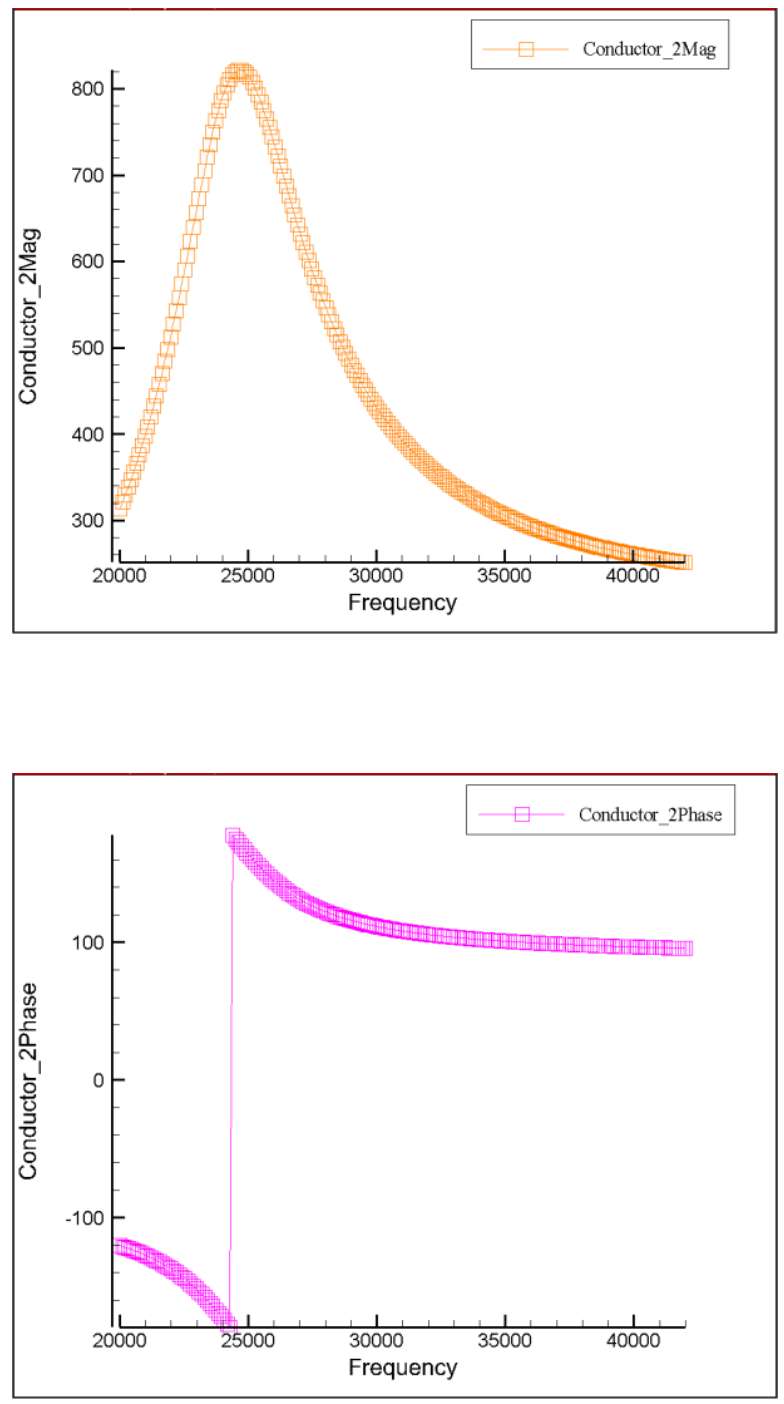

Figure 23. Frequency Response at $1.4 \mu \mathrm{m}$ 
2. Gap Size: $1.3 \mu \mathrm{m}$ and DC Bias Voltage: 5.5V
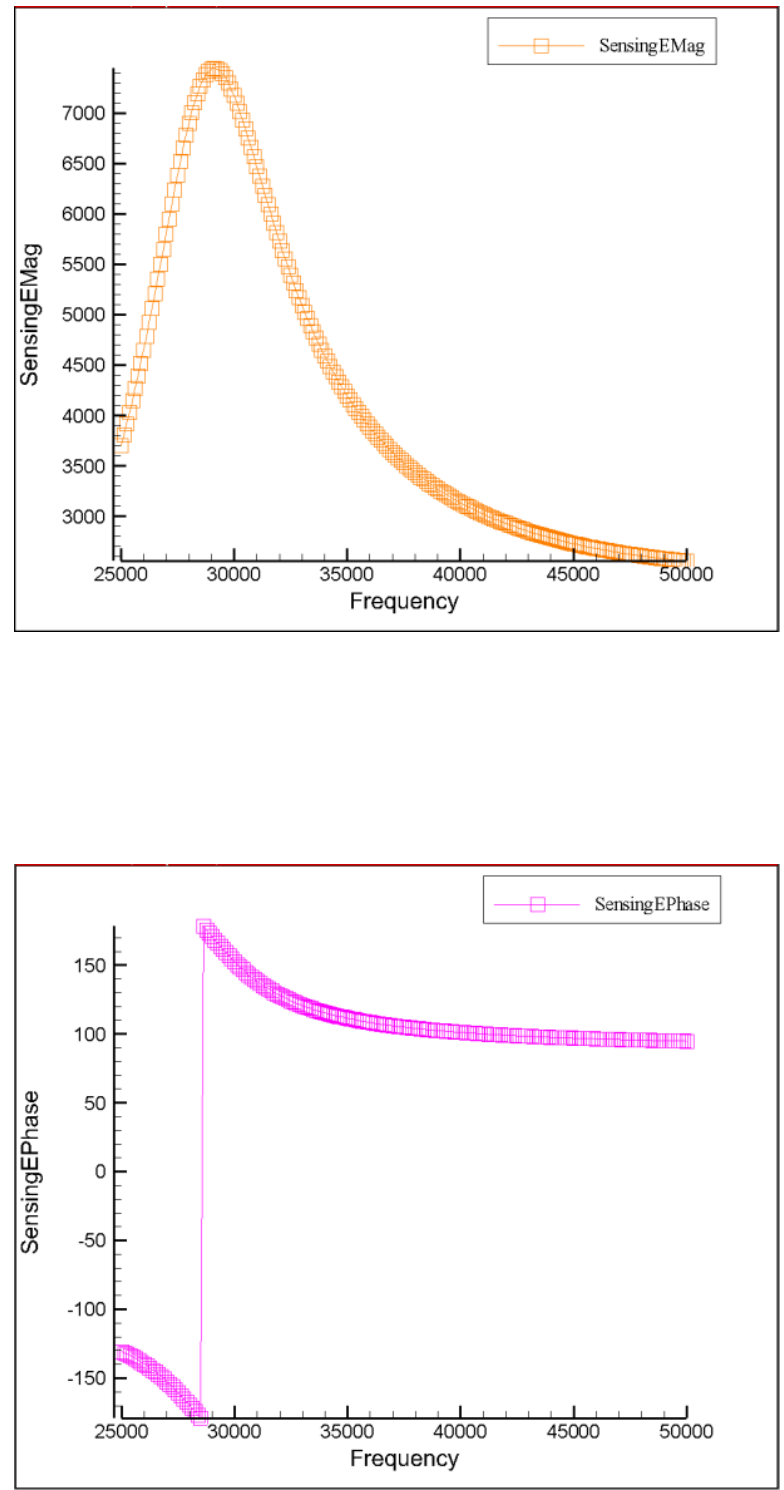

Figure 24. Frequency Response at $1.3 \mu \mathrm{m}$ 
3. Gap Size: $1 \mu \mathrm{m}$ and DC Bias Voltage: $3 \mathrm{~V}$
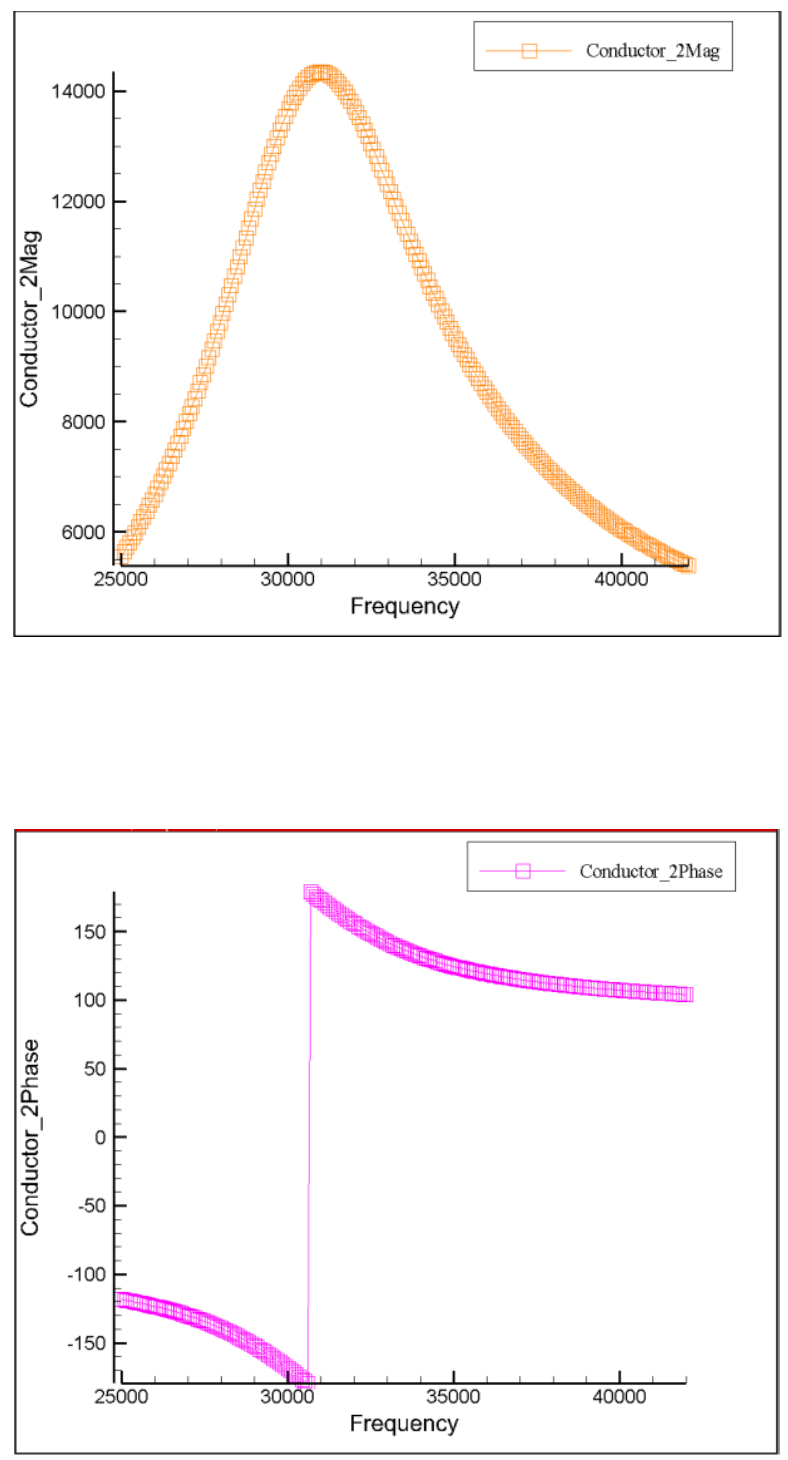

Figure 25. Frequency Response at $\mathbf{1} \boldsymbol{\mu m}$ 
4. Gap Size: $0.8 \mu \mathrm{m}$ and DC Bias Voltage: $2.2 \mathrm{~V}$
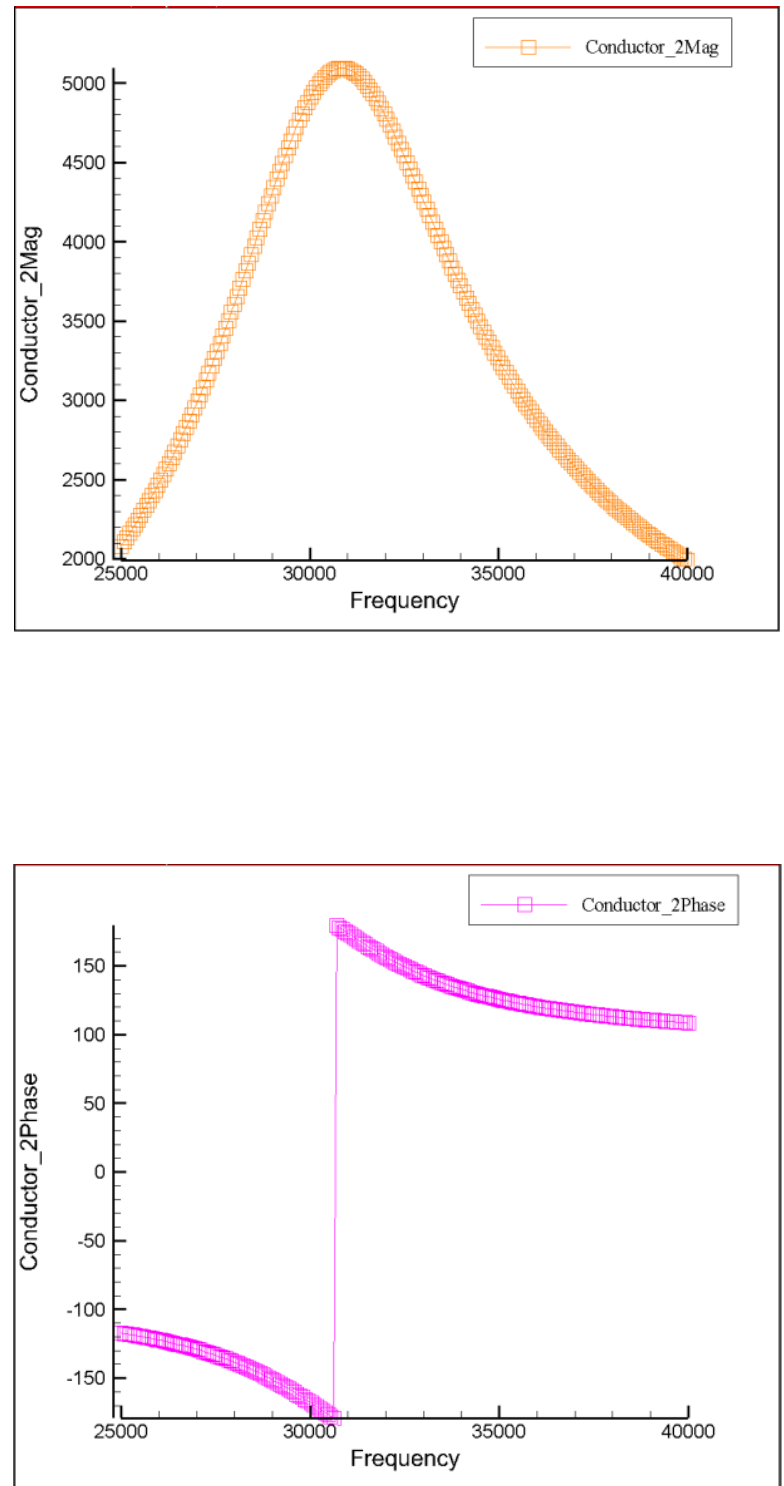

Figure 26. Frequency Response at $0.8 \mu \mathrm{m}$ 
5. Gap Size: $0.7 \mu \mathrm{m}$ and DC Bias Voltage: $1.8 \mathrm{~V}$
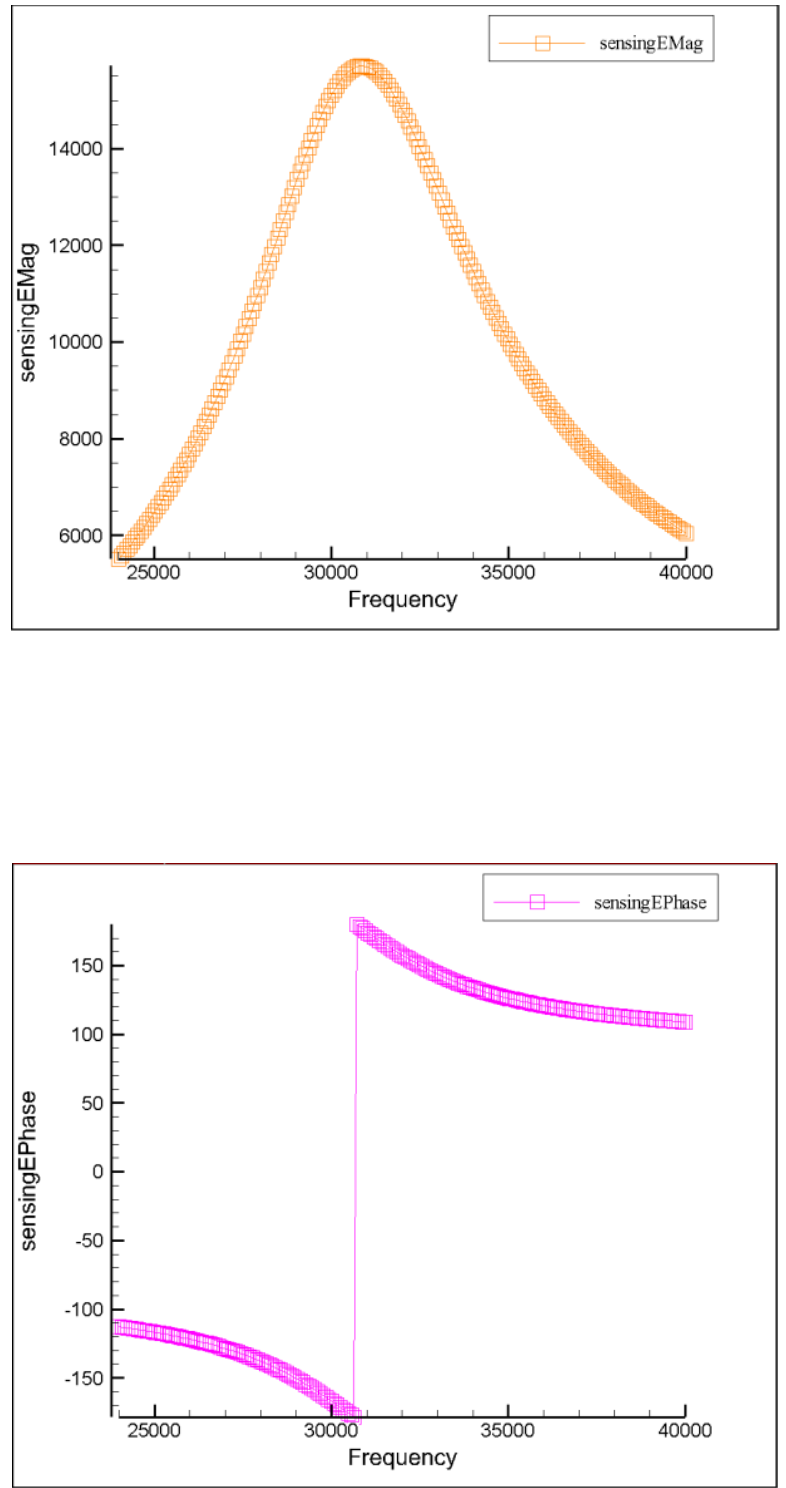

Figure 27. Frequency Response at $0.7 \mu \mathrm{m}$ 
6. Gap Size: $0.6 \mu \mathrm{m}$ and DC Bias Voltage: $0.8 \mathrm{~V}$
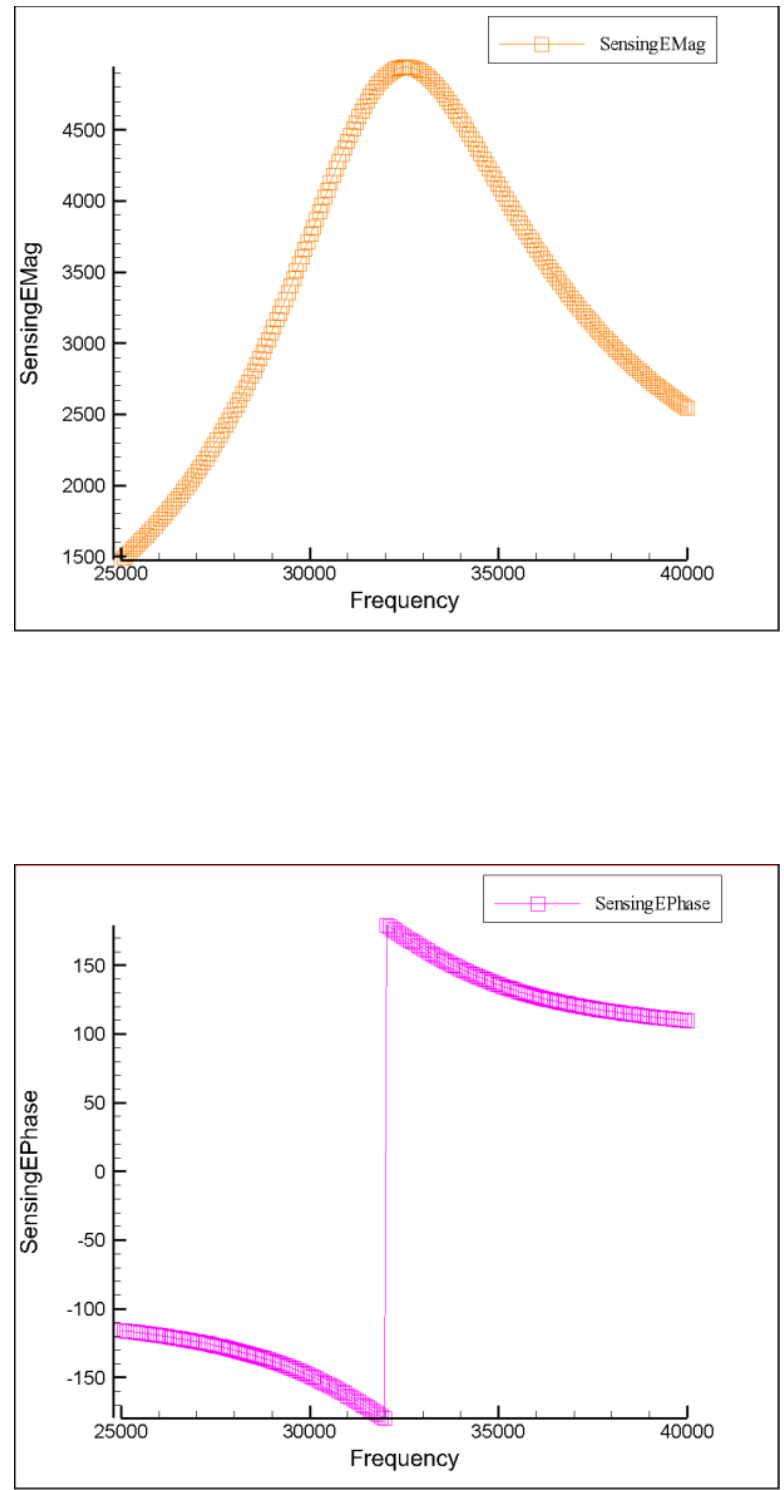

Figure 28. Frequency Response at $0.6 \mu \mathrm{m}$ 
7. Gap Size: $0.5 \mu \mathrm{m}$ and DC Bias Voltage: 0.7V
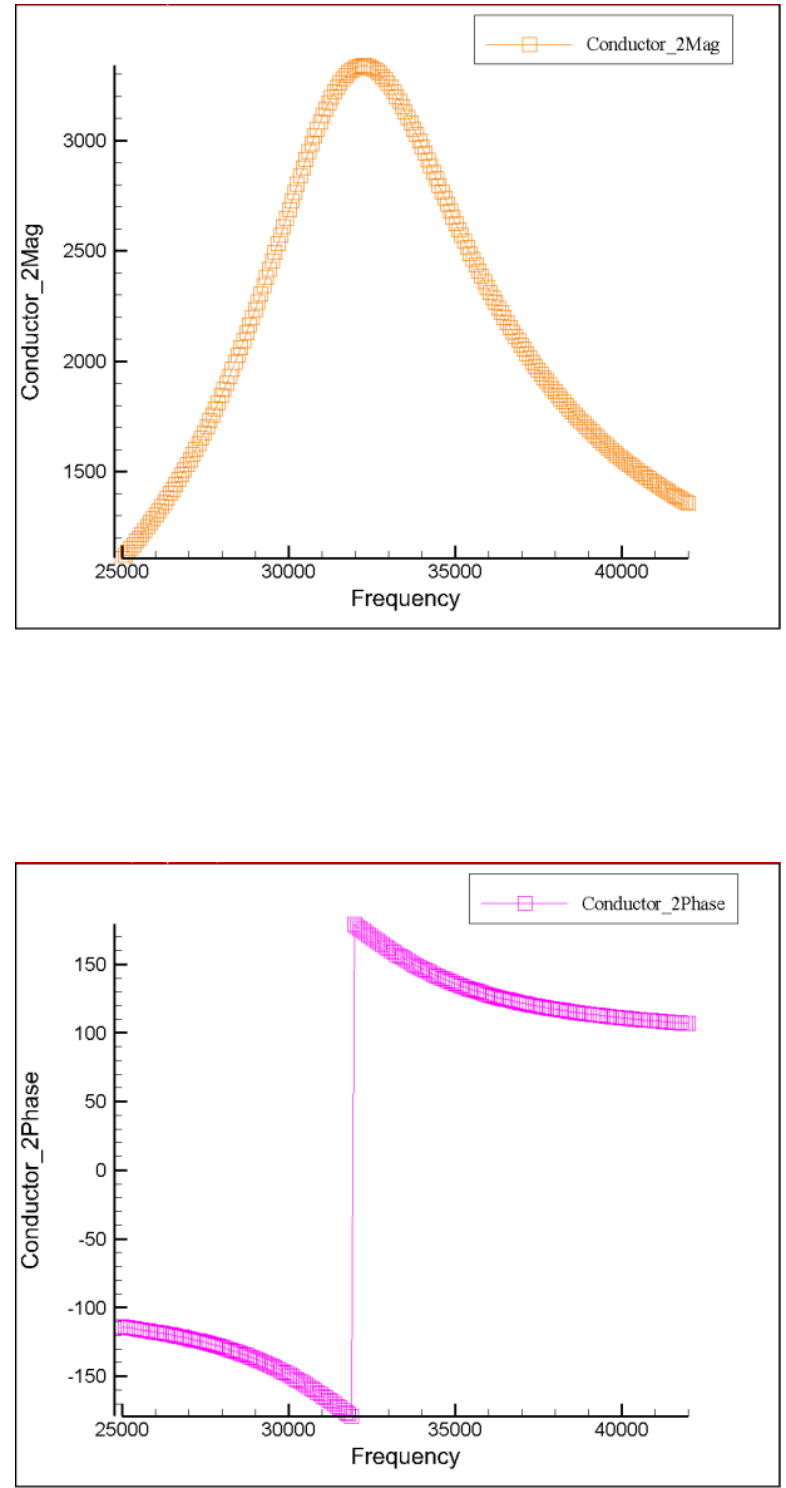

Figure 29. Frequency Response at $0.5 \mu \mathrm{m}$ 
8. Gap Size: $0.4 \mu \mathrm{m}$ and DC Bias Voltage: $0.5 \mathrm{~V}$
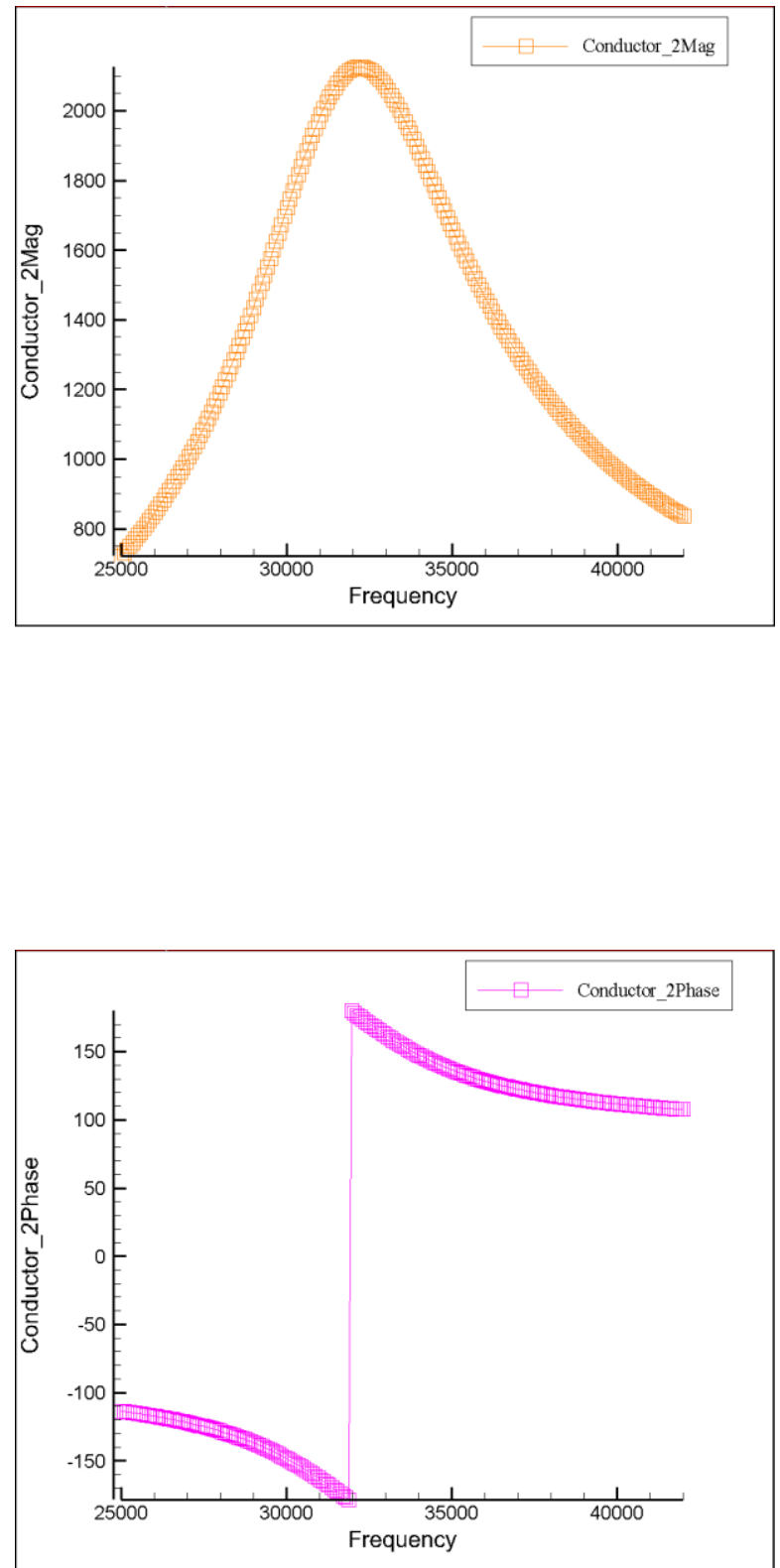

Figure 30. Frequency Response at $0.4 \mu \mathrm{m}$ 
9. Gap Size: $0.2 \mu \mathrm{m}$ and DC Bias Voltage: $0.12 \mathrm{~V}$
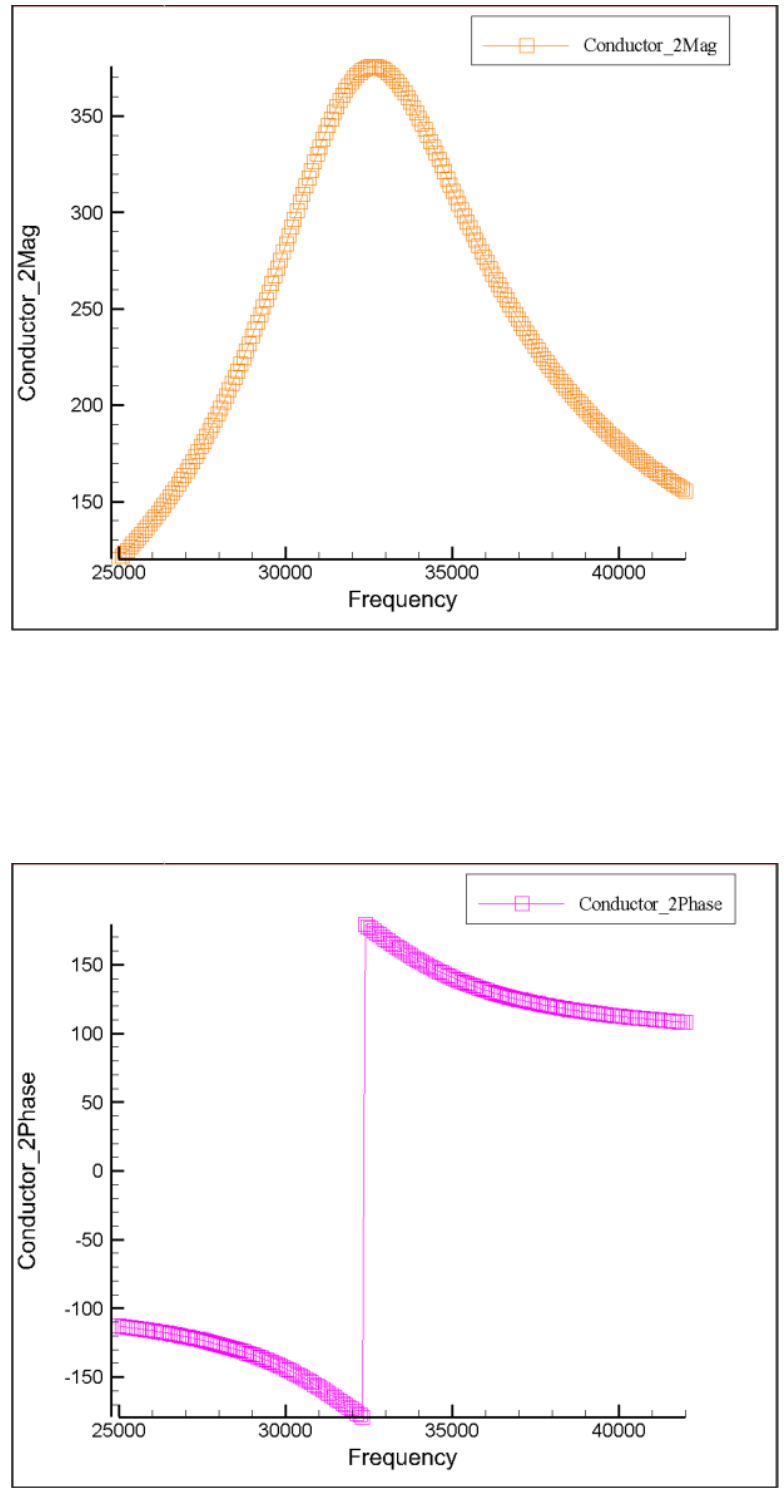

Figure 31. Frequency Response at $0.2 \mu \mathrm{m}$ 
10. Gap Size: $0.1 \mu \mathrm{m}$ and DC Bias Voltage: 0.03V
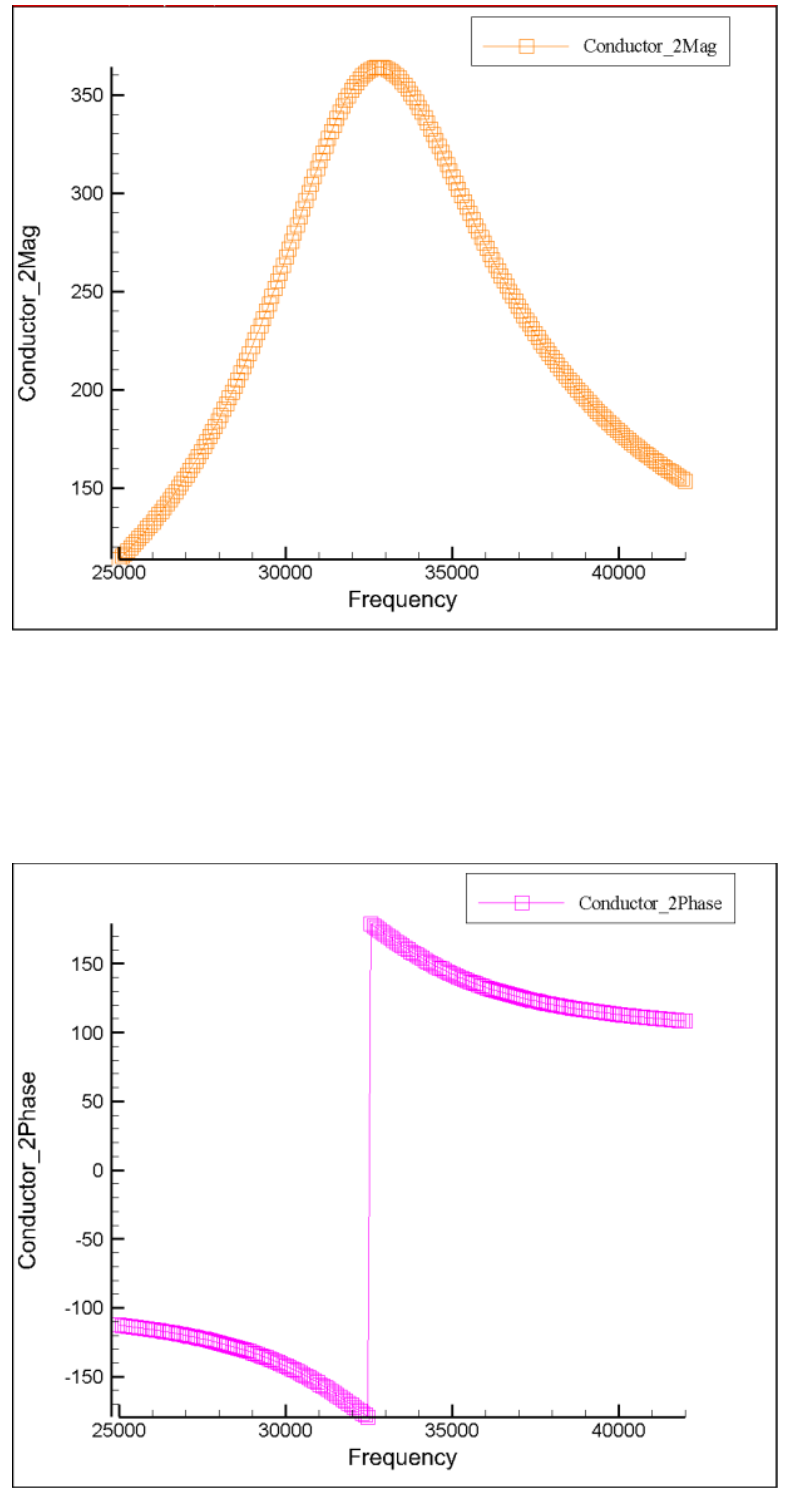

Figure 32. Frequency Response at $0.1 \mu \mathrm{m}$ 
11. Gap Size: $80 \mathrm{~nm}$ and DC Bias Voltage: $0.02 \mathrm{~V}$
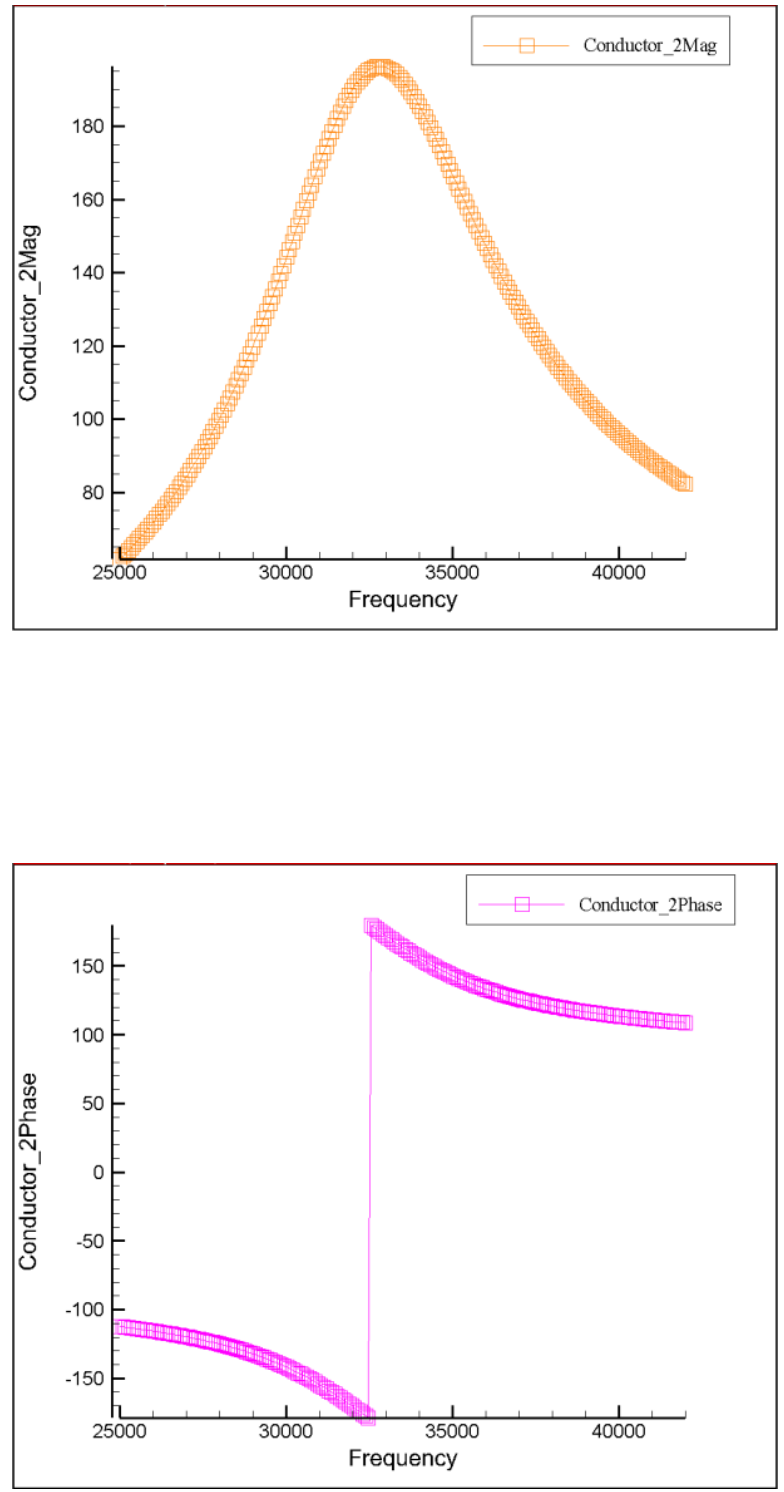

Figure 33. Frequency Response at 80nm 